Network Working Group

Request for Comments: 1628

Category: Standards Track
J. Case, Editor

SNMP Research, Incorporated

\title{
UPS Management Information Base
}

Status of this Memo

This document specifies an Internet standards track protocol for the Internet community, and requests discussion and suggestions for improvements. Please refer to the current edition of the "Internet Official Protocol standards" (STD 1) for the standardization state and status of this protocol. Distribution of this memo is unlimited.

Table of Contents

1. Introduction $\ldots \ldots \ldots \ldots \ldots \ldots \ldots \ldots \ldots \ldots \ldots \ldots \ldots \ldots \ldots$

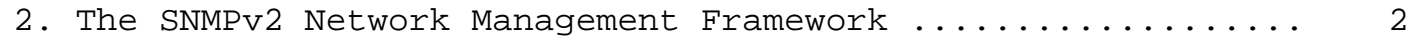

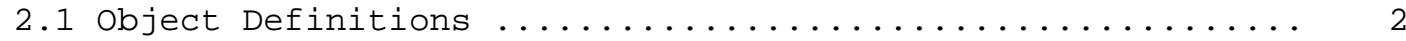

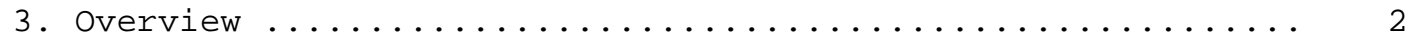

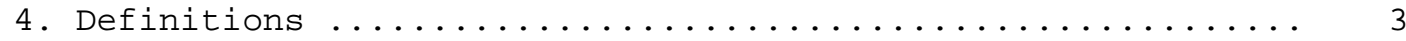

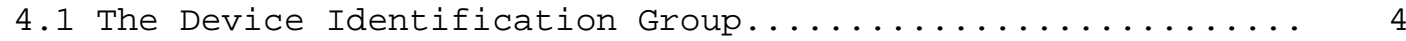

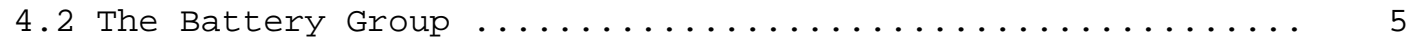

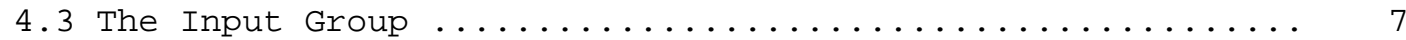

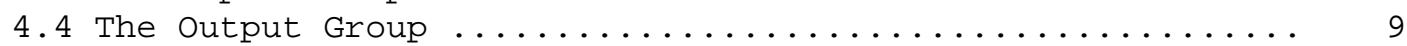

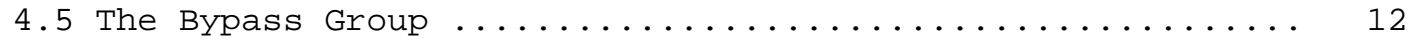

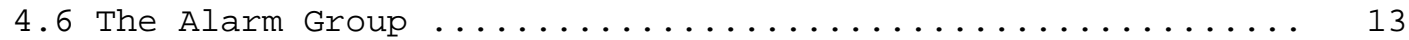

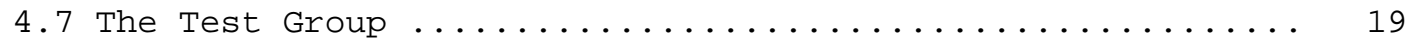

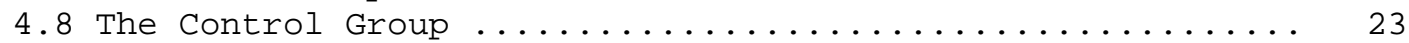

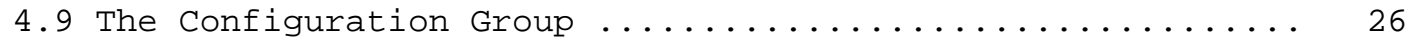

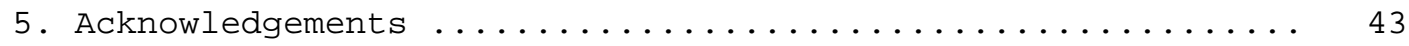

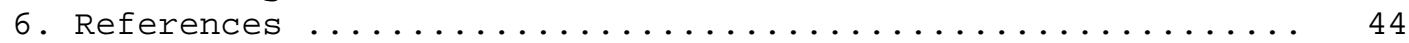

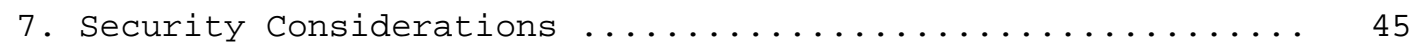

8. Author's Address ............................ 45

\section{Introduction}

This memo defines a portion of the Management Information Base (MIB) for use with network management protocols in the Internet community. In particular, it defines objects for managing uninterruptible power supply (UPS) systems. 
2. The SNMPv2 Network Management Framework

The SNMPv2 Network Management Framework consists of four major components. They are:

- RFC 1442 which defines the SMI, the mechanisms used for describing and naming objects for the purpose of management.

- STD 17, RFC 1213 defines MIB-II, the core set of managed objects for the Internet suite of protocols.

- RFC 1445 which defines the administrative and other architectural aspects of the framework.

- RFC 1448 which defines the protocol used for network access to managed objects.

The Framework permits new objects to be defined for the purpose of experimentation and evaluation.

\subsection{Object Definitions}

Managed objects are accessed via a virtual information store, termed the Management Information Base or MIB. Objects in the MIB are defined using the subset of Abstract Syntax Notation One (ASN.1) defined in the SMI. In particular, each object type is named by an OBJECT IDENTIFIER, an administratively assigned name. The object type together with an object instance serves to uniquely identify a specific instantiation of the object. For human convenience, we often use a textual string, termed the descriptor, to refer to the object type.

\section{Overview}

This document defines the managed objects for Uninterruptible Power Supplies which are to be manageable via the Simple Network Management Protocol (SNMP). 
4. Definitions

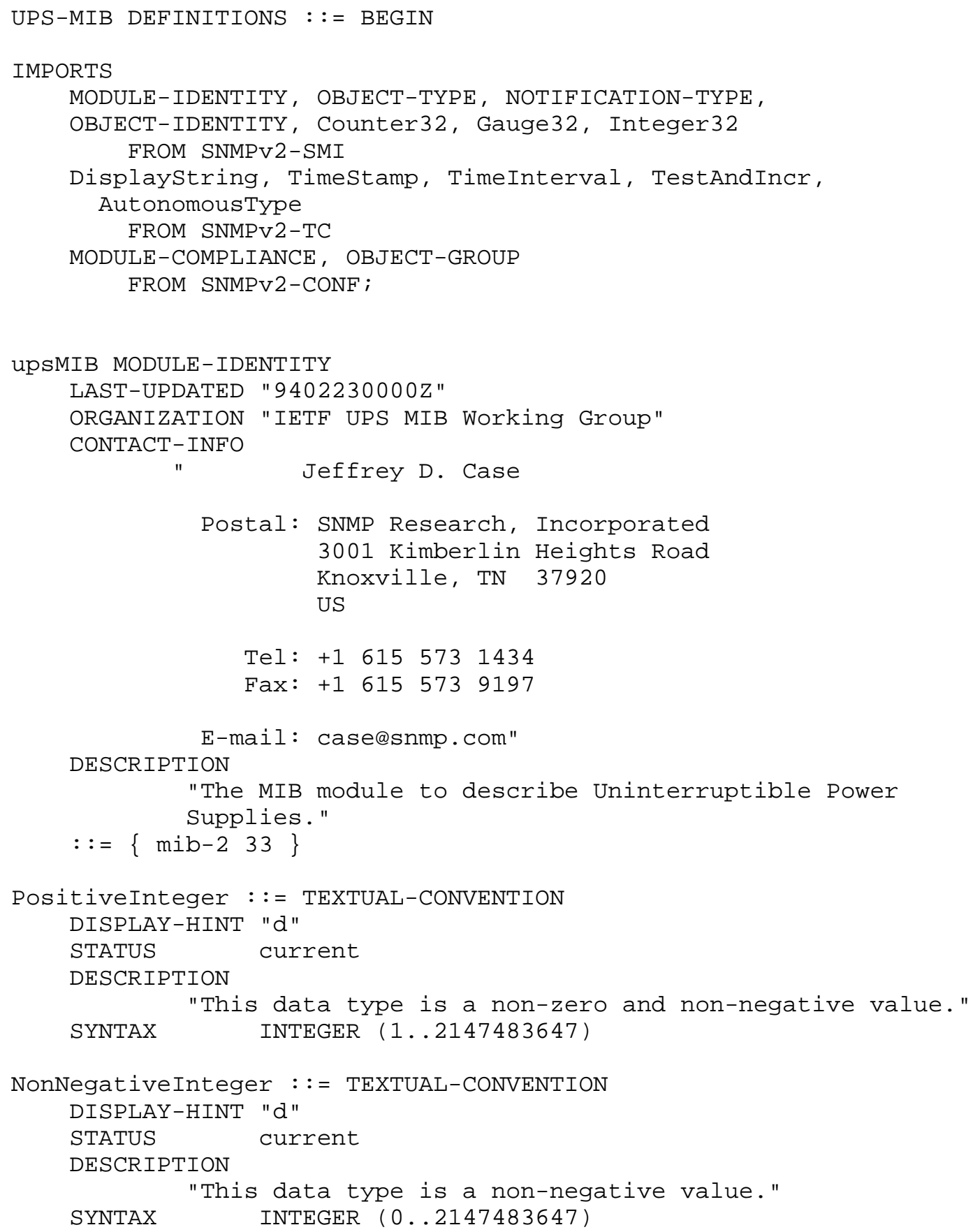




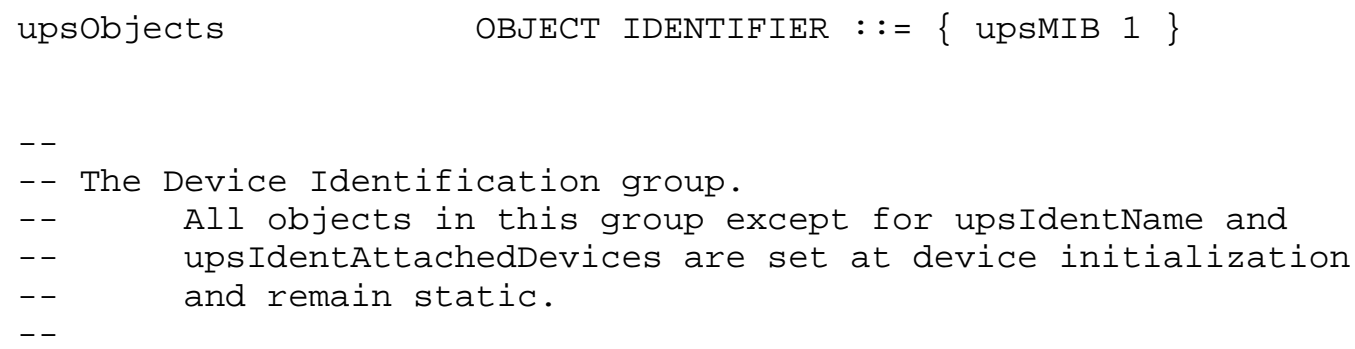




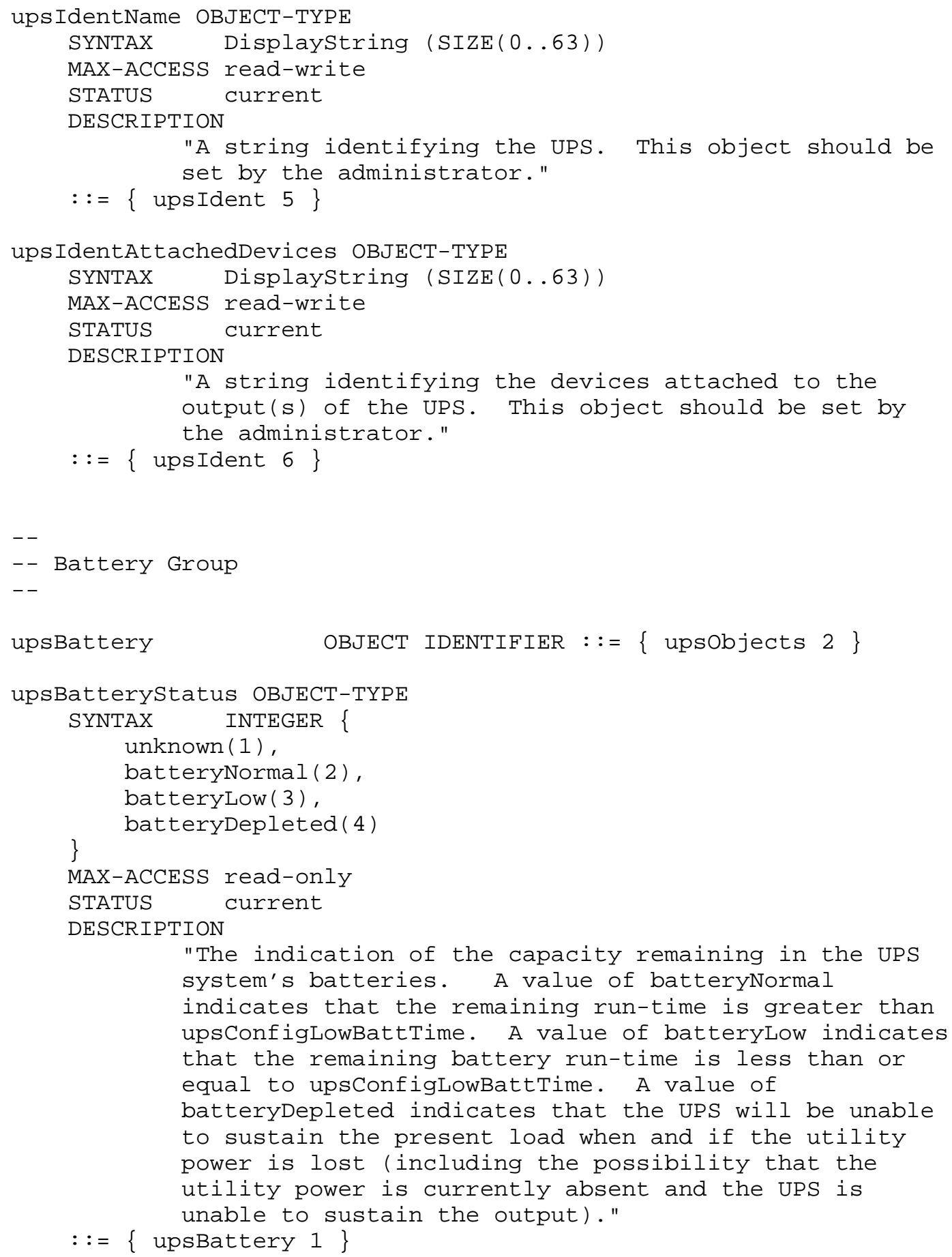




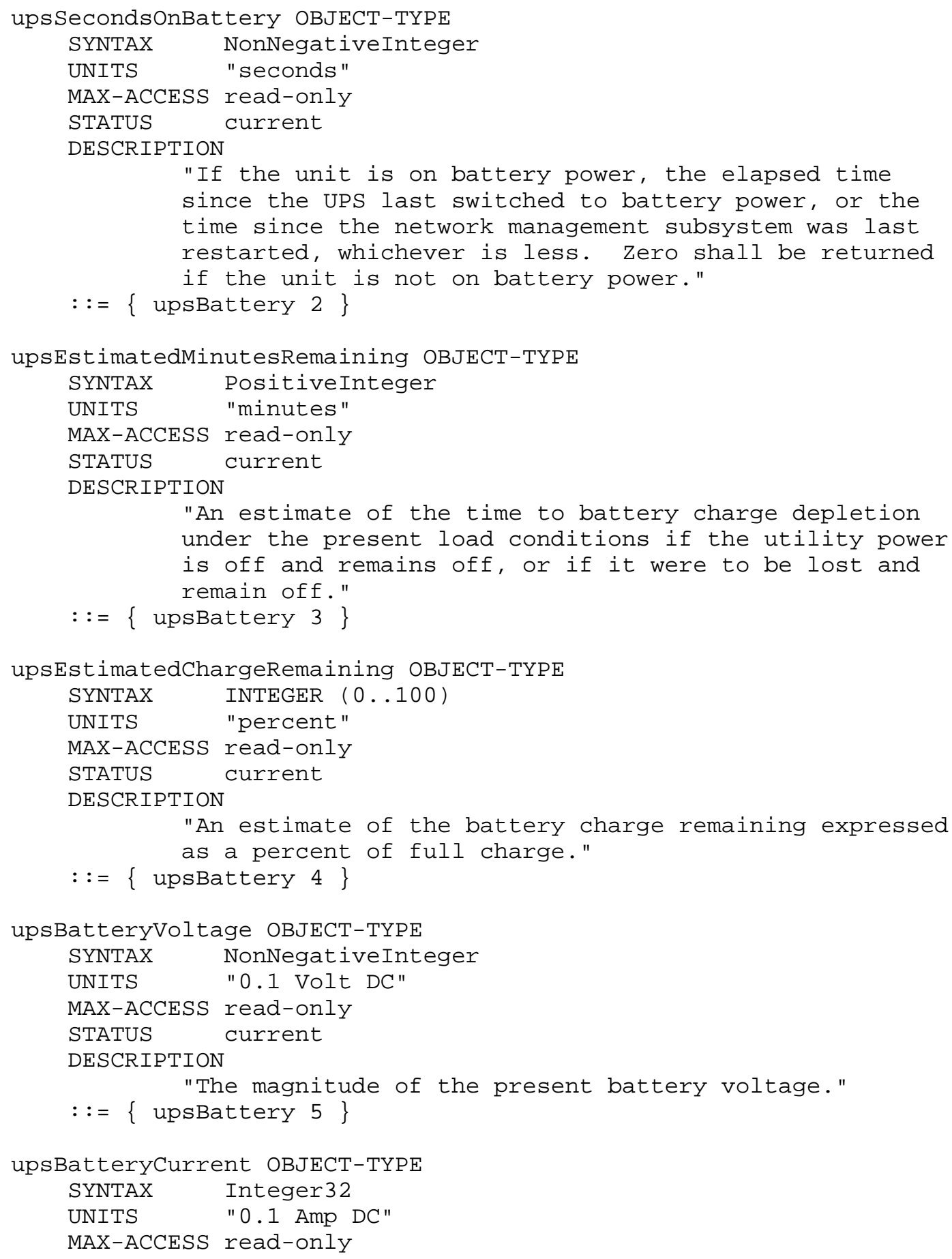




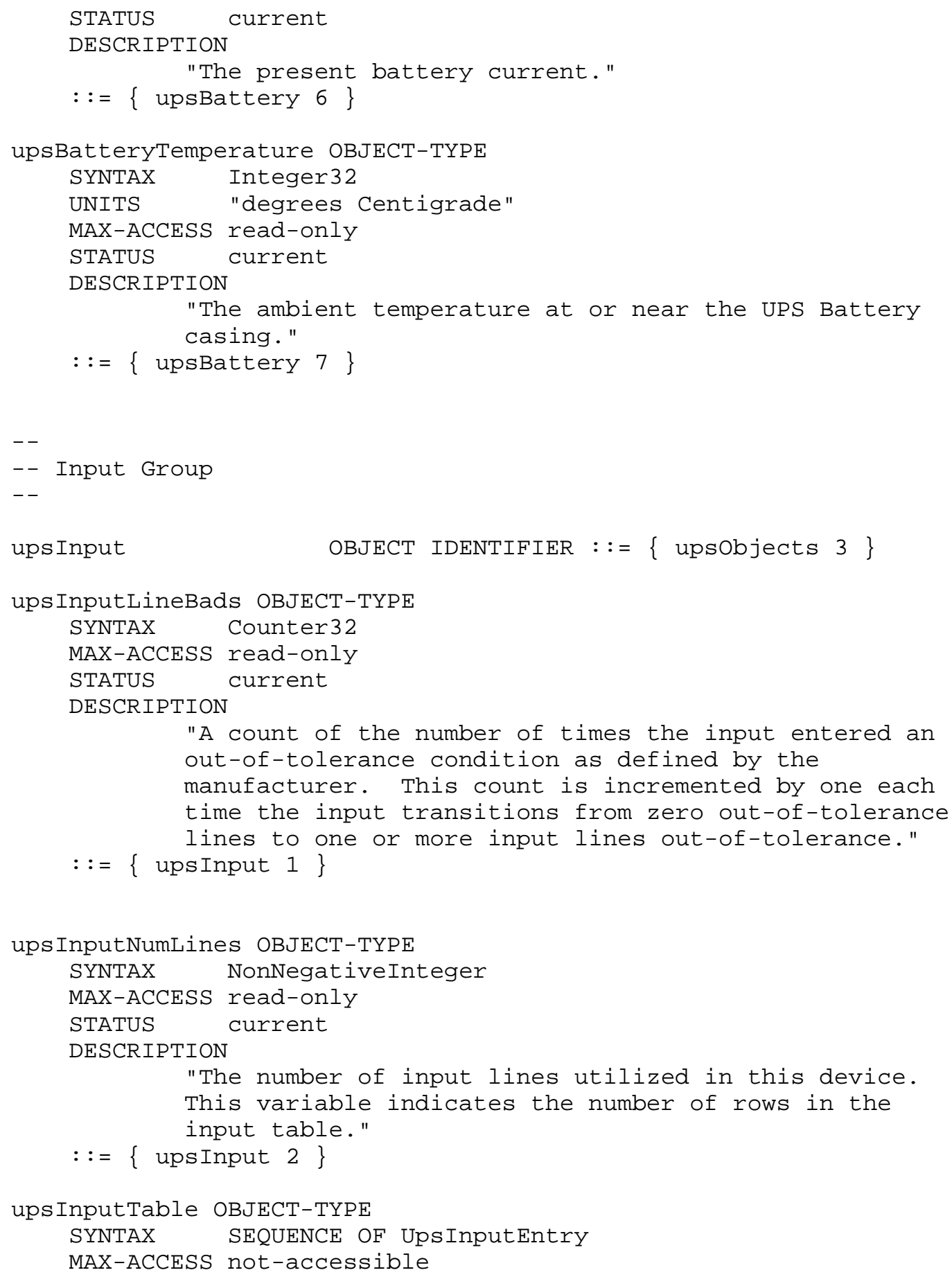




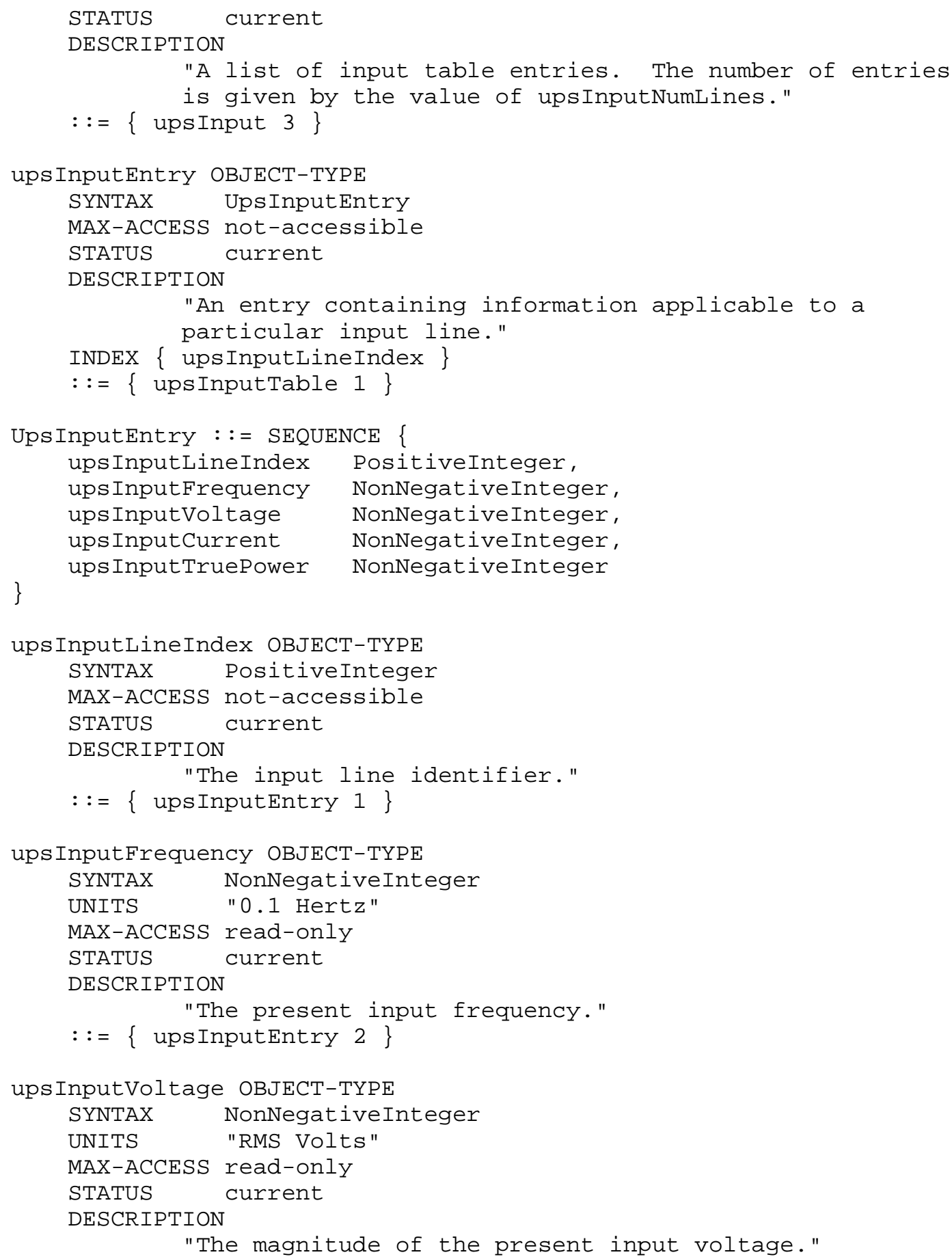




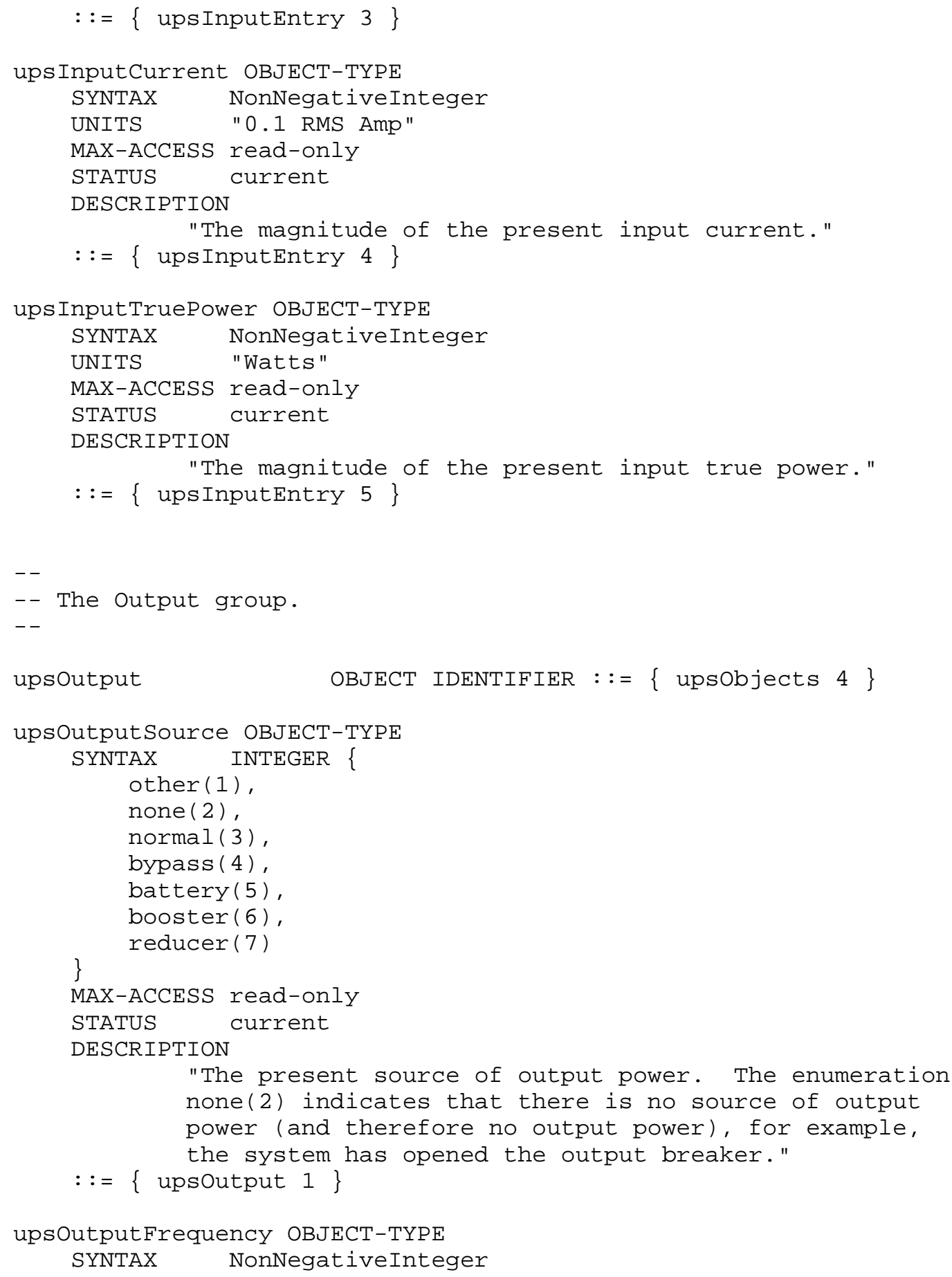




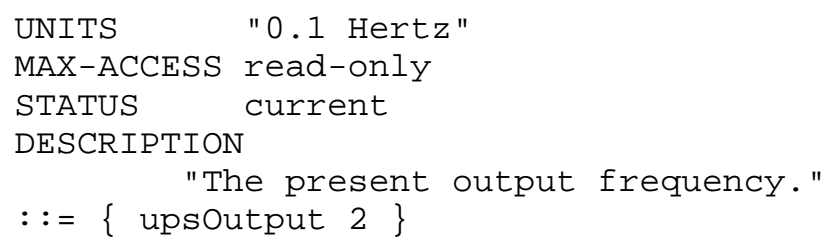




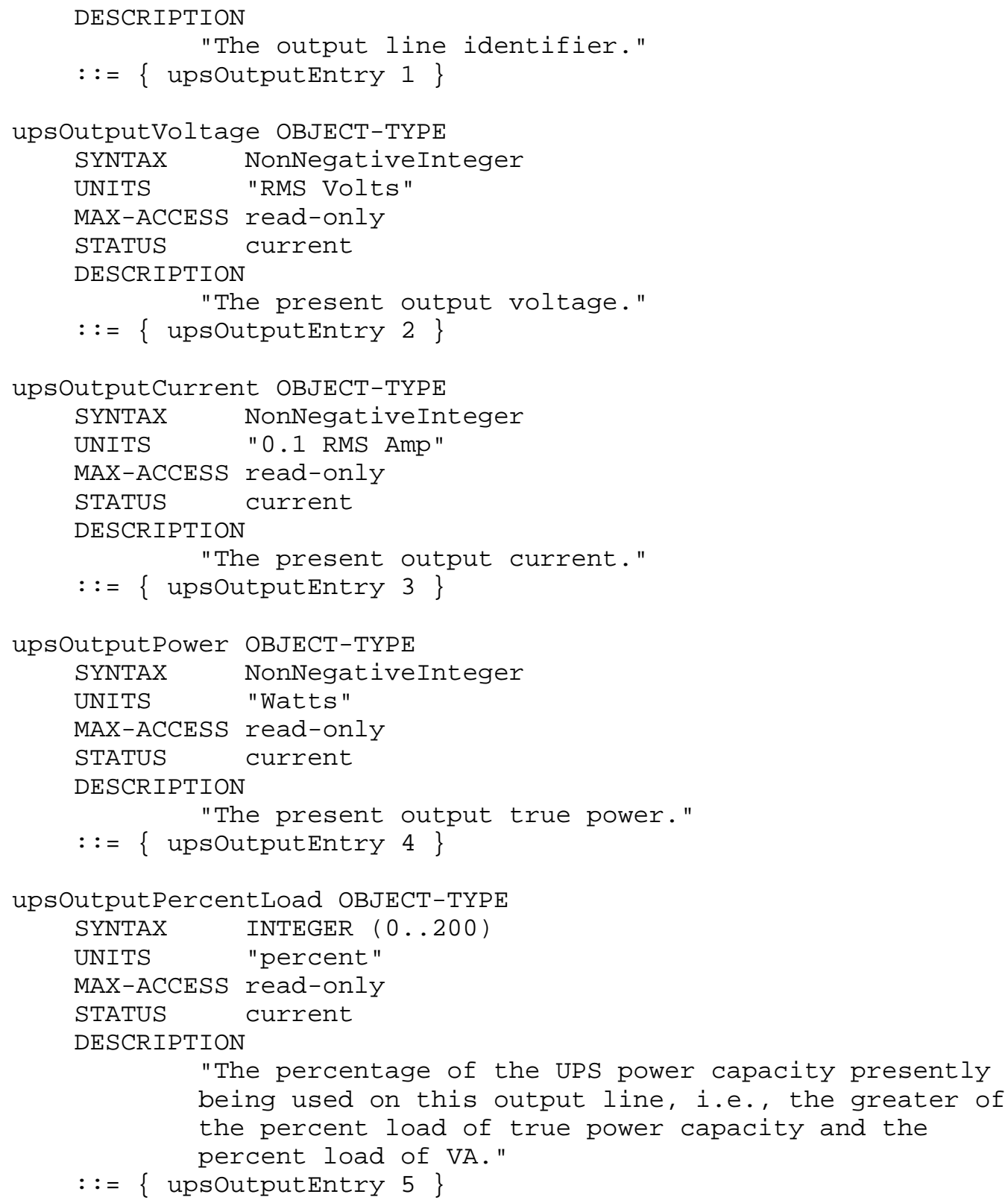




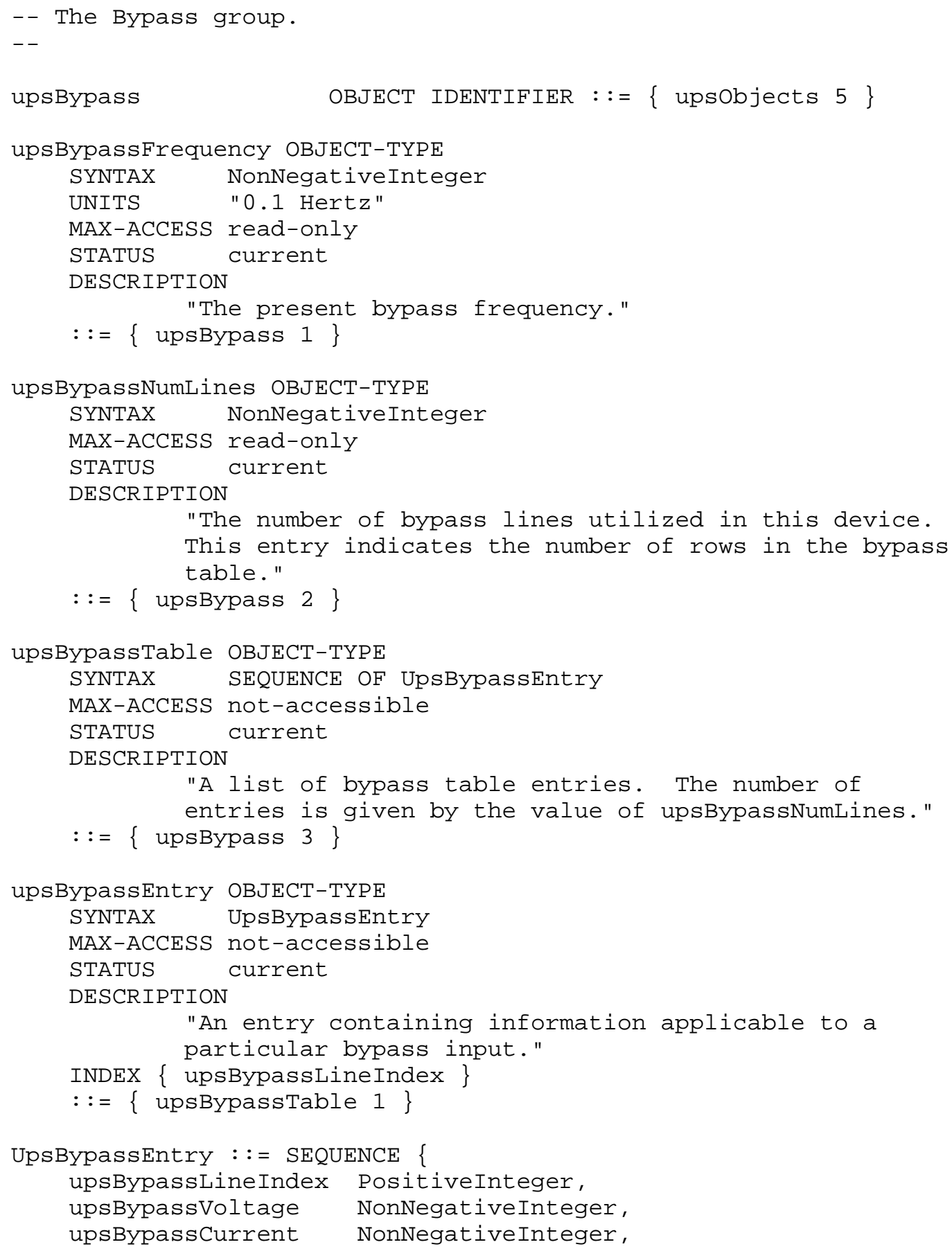




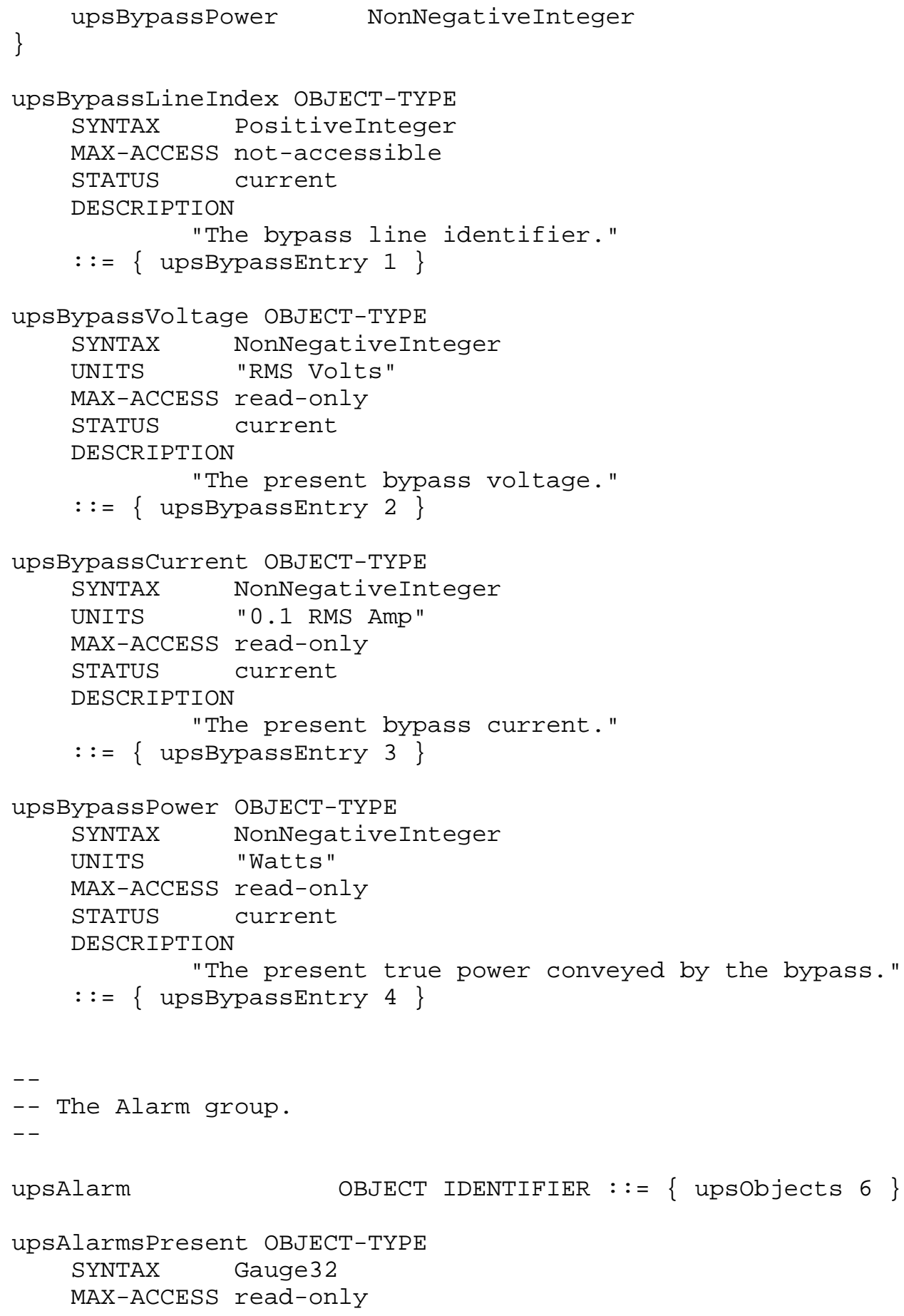




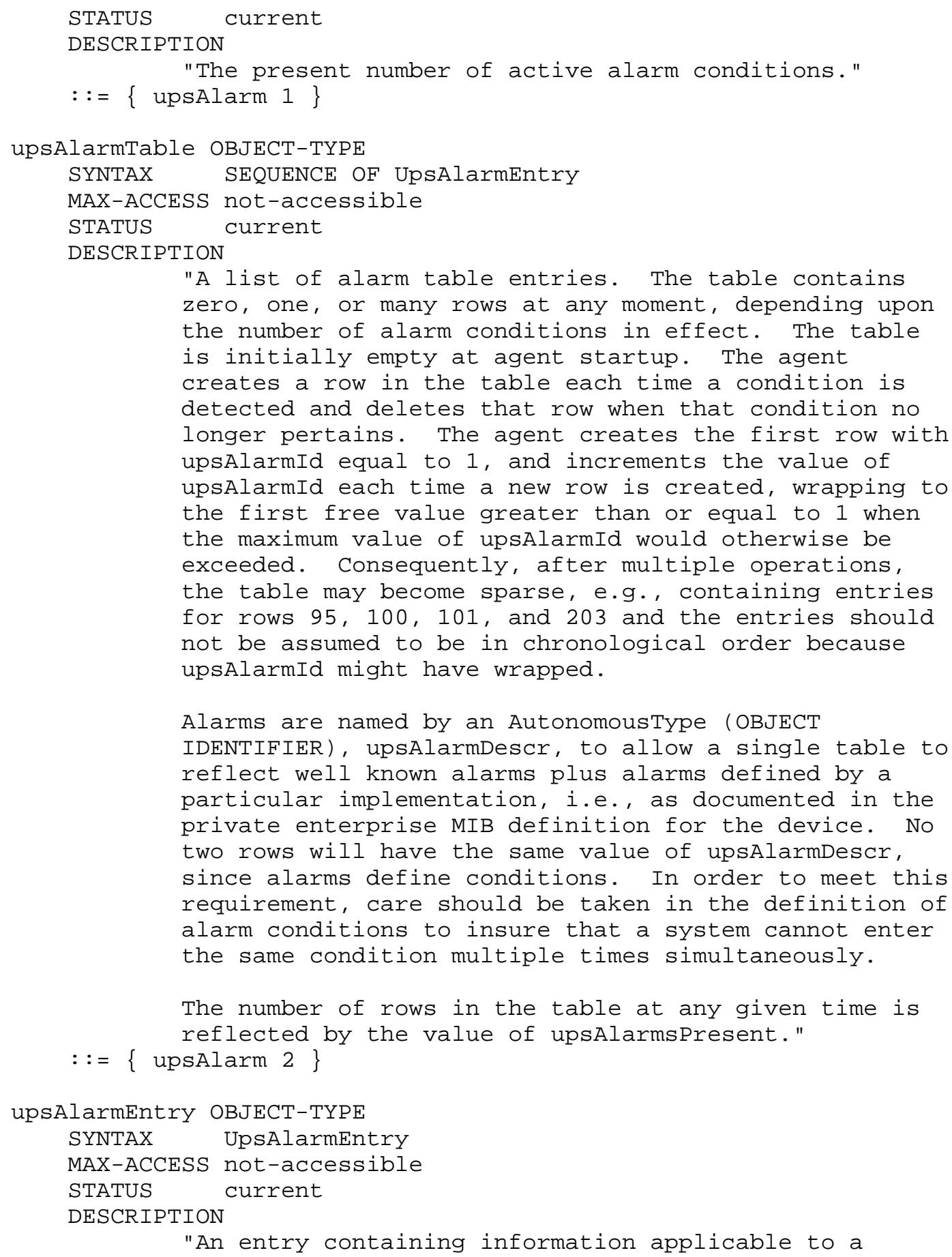




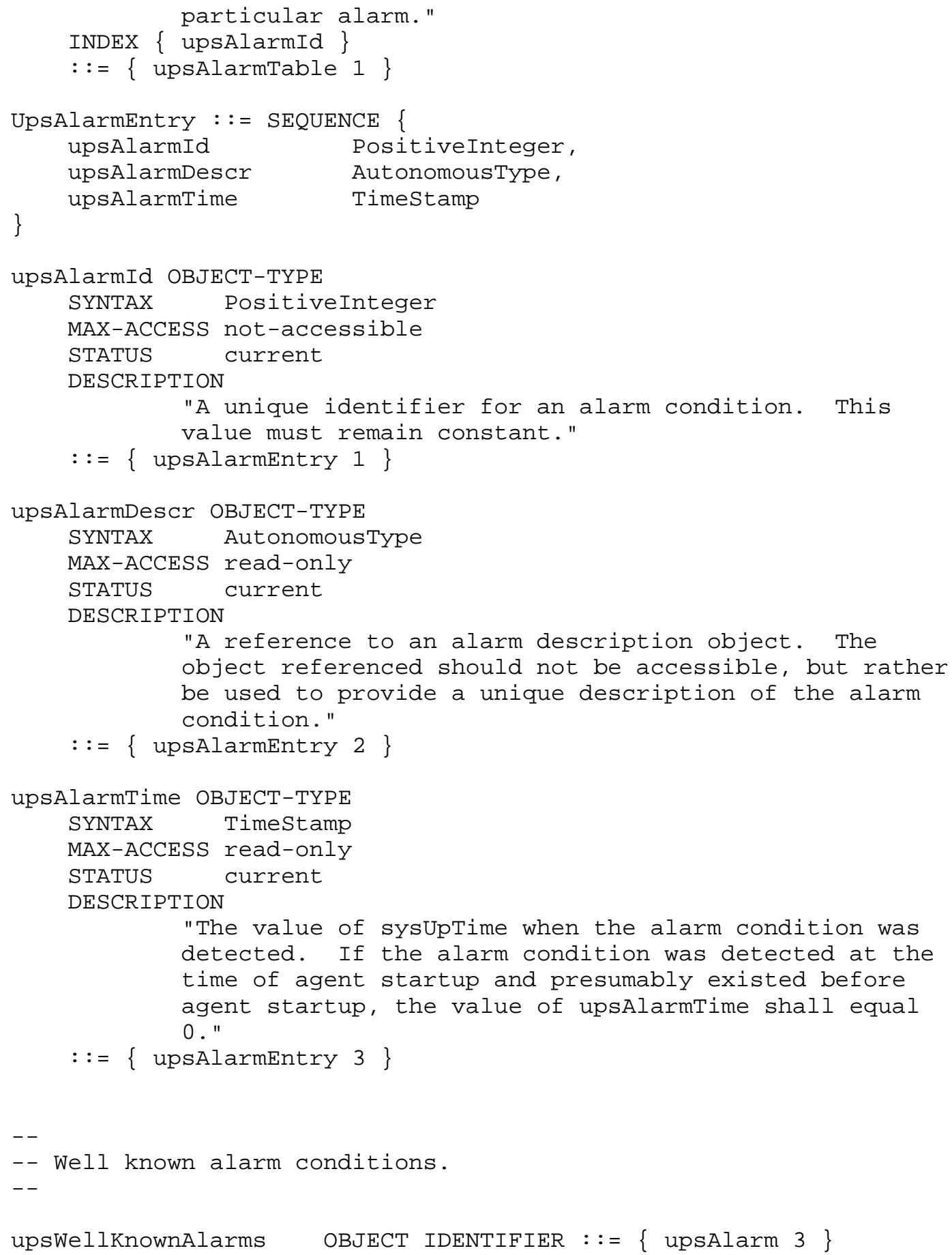




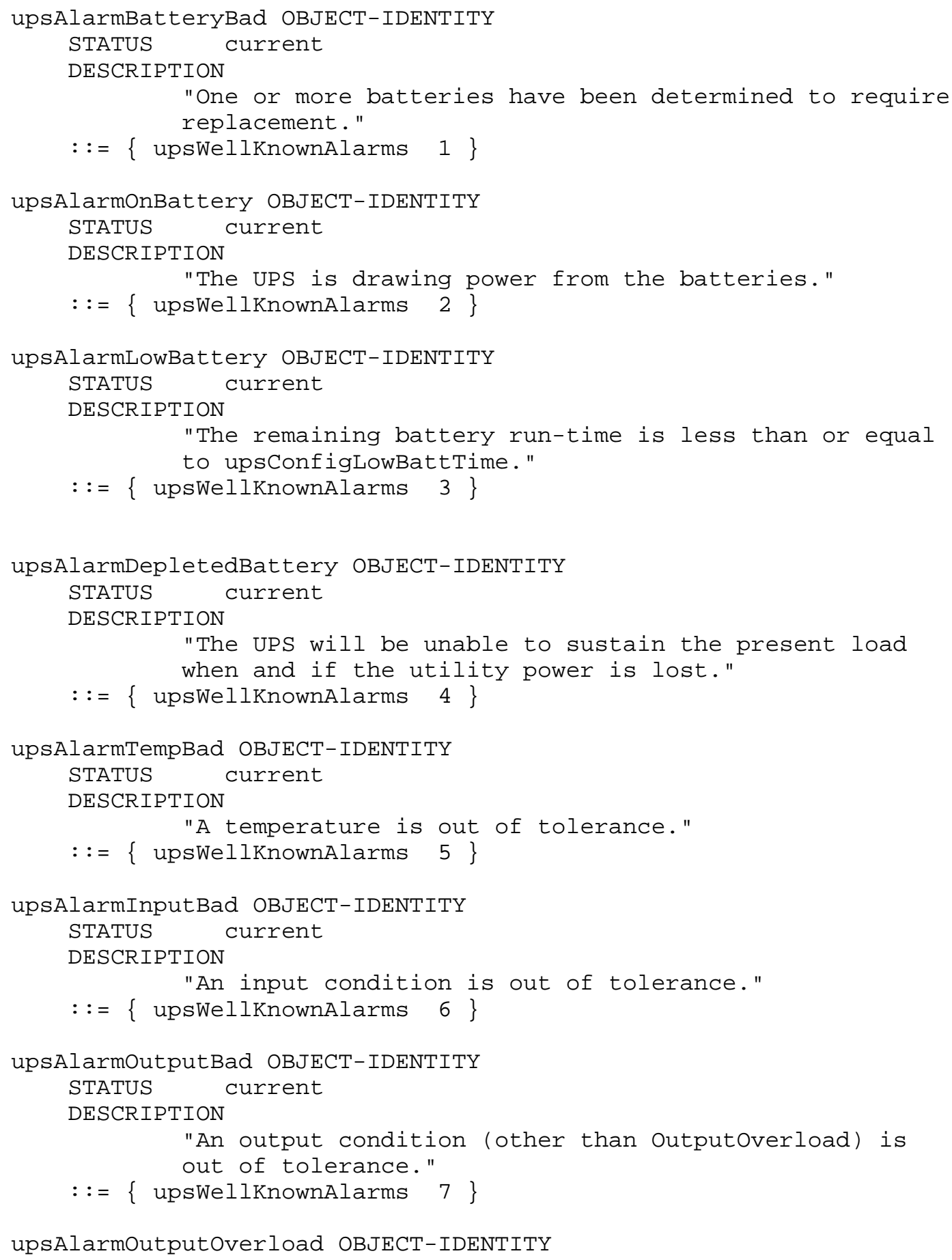




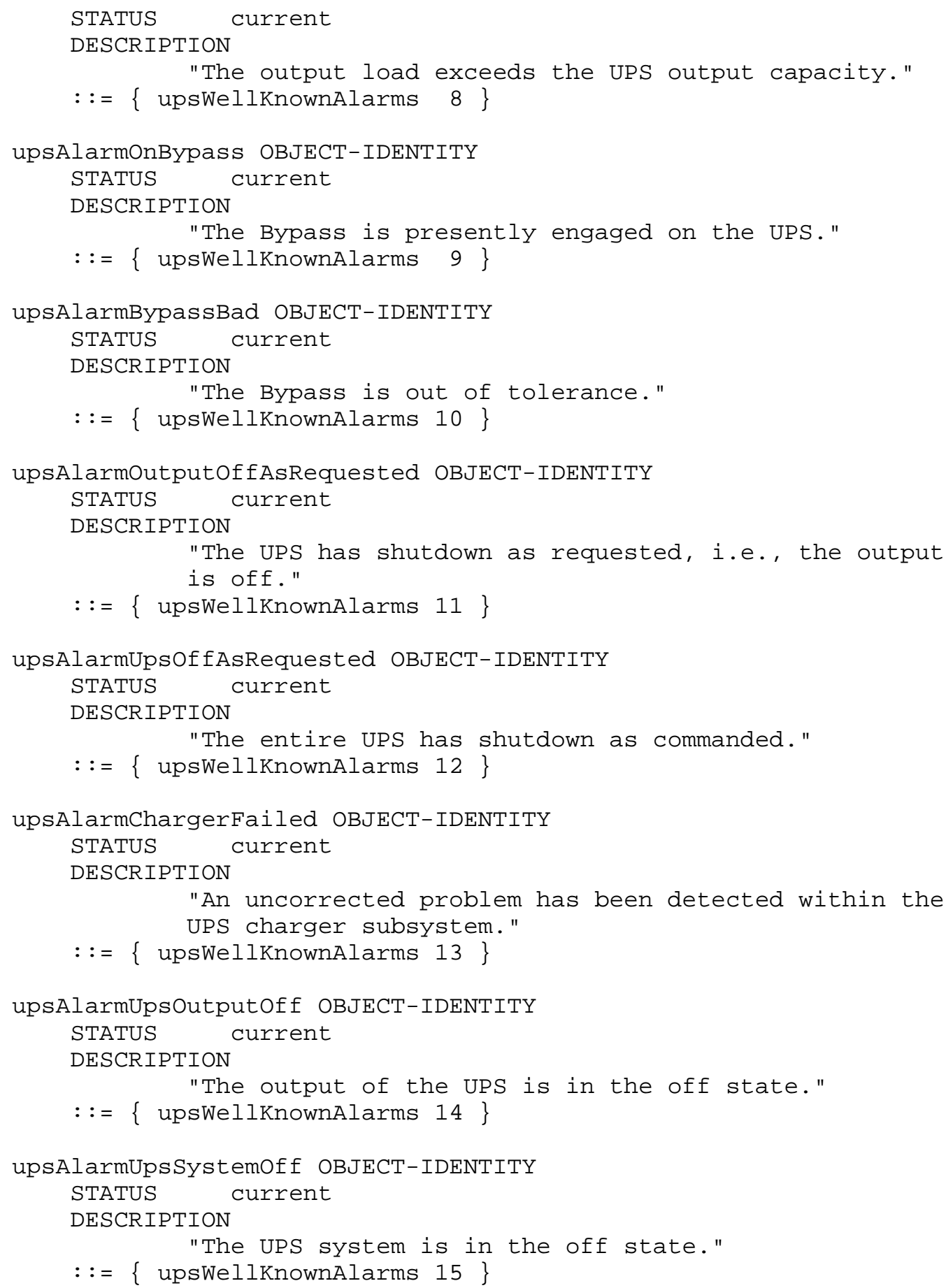




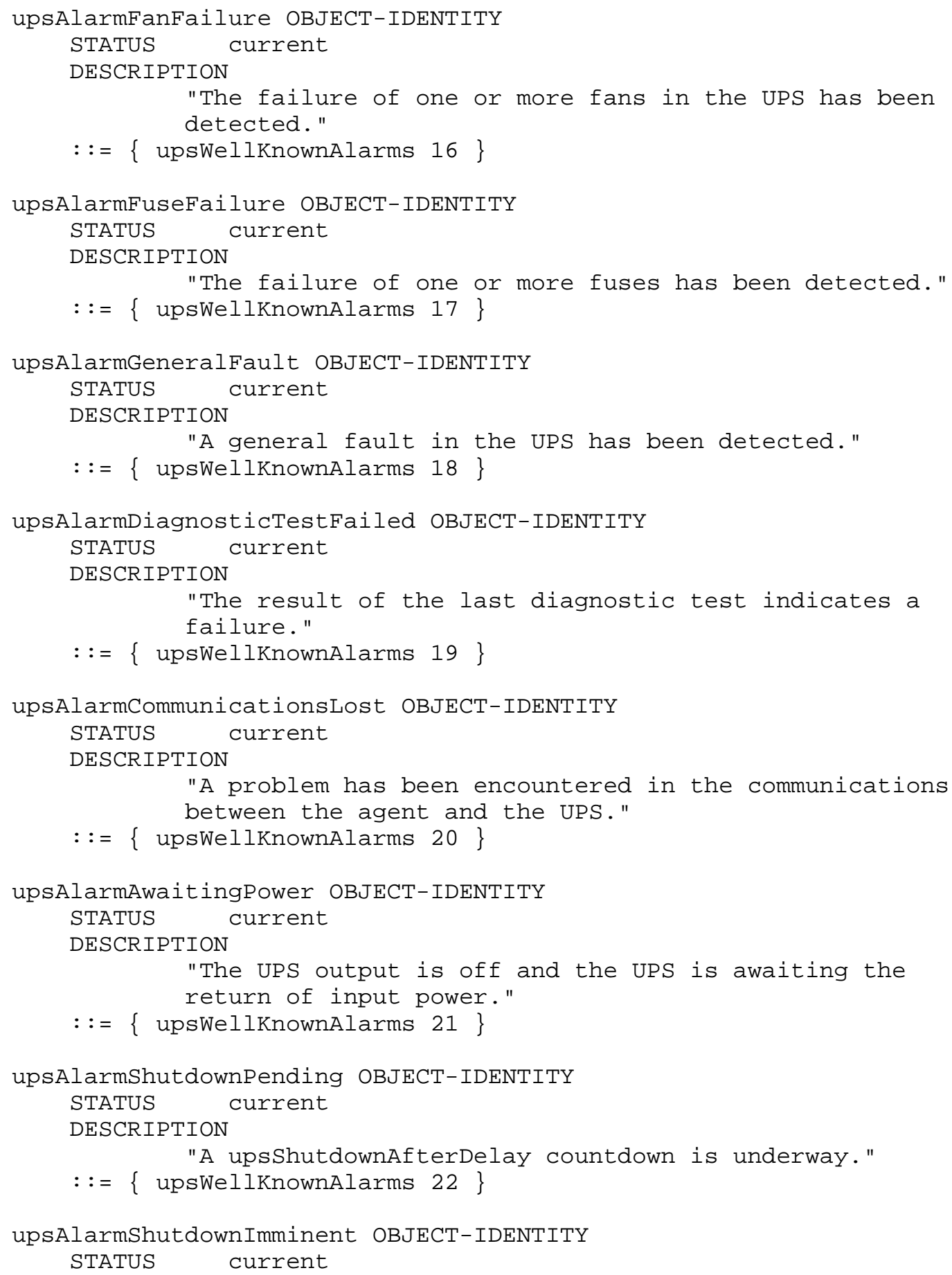




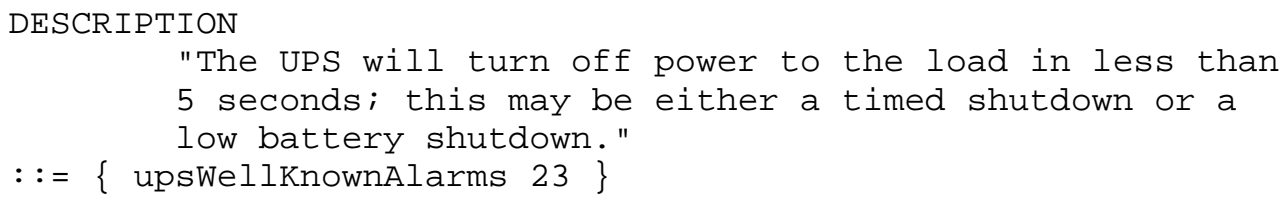




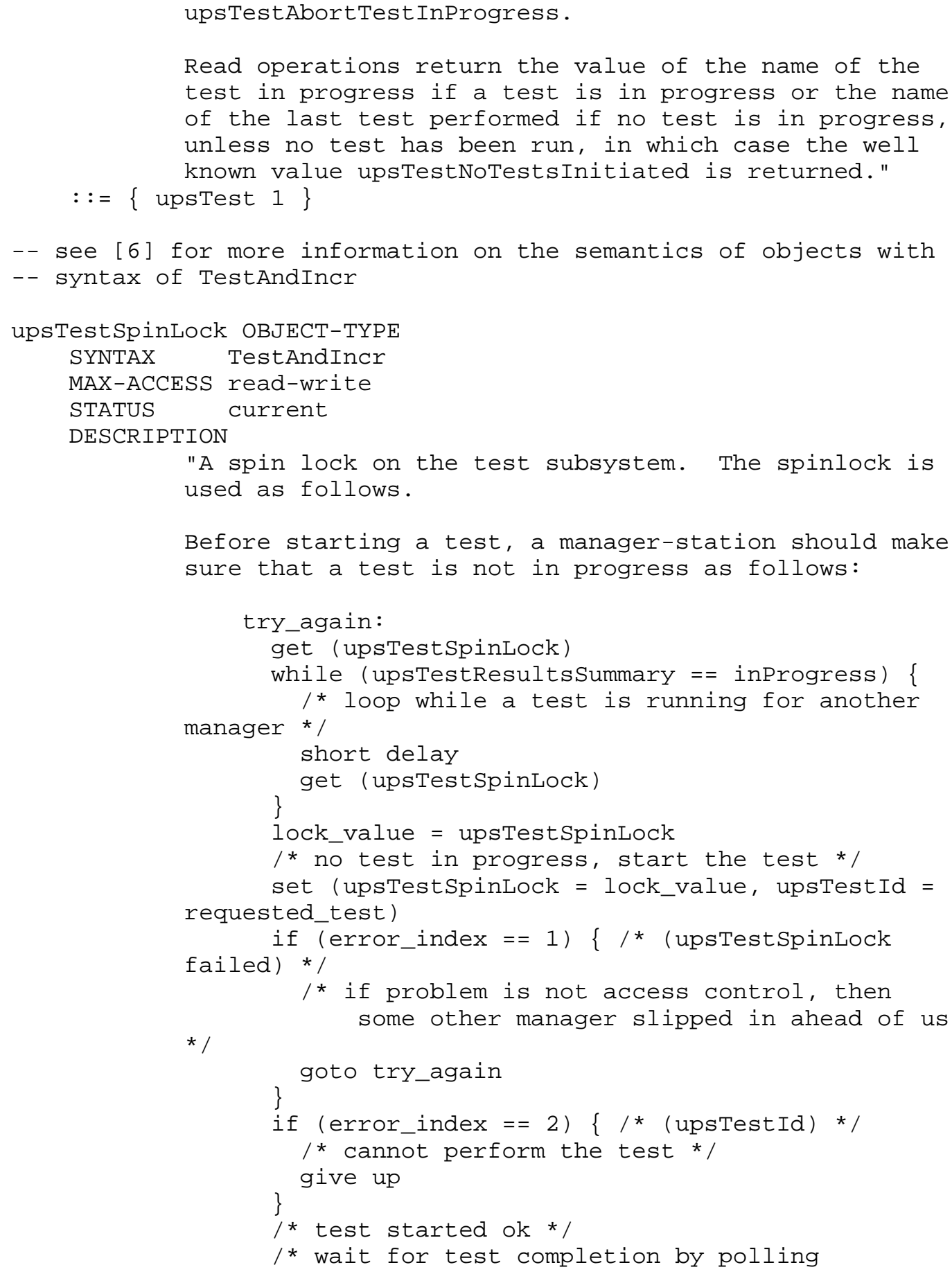




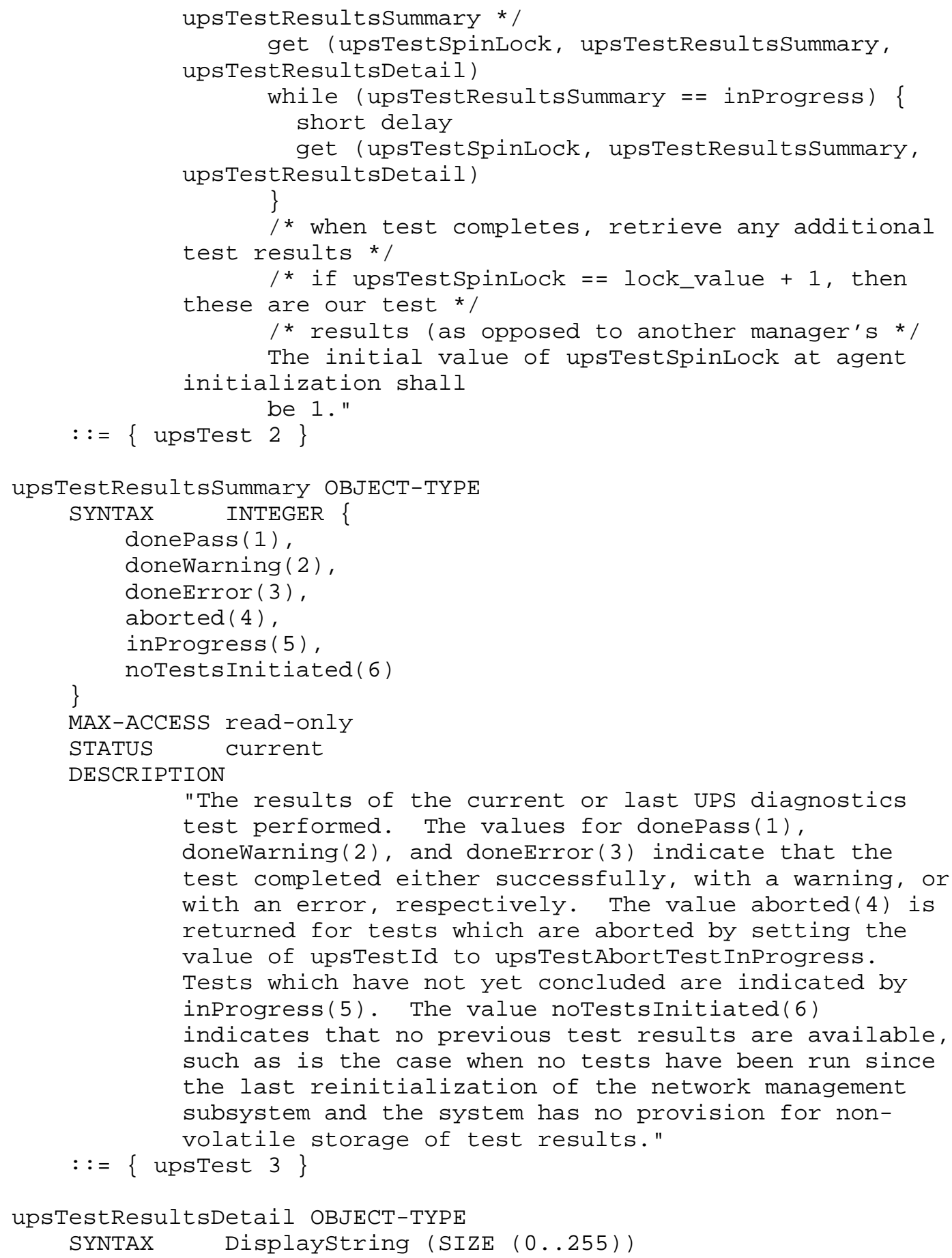




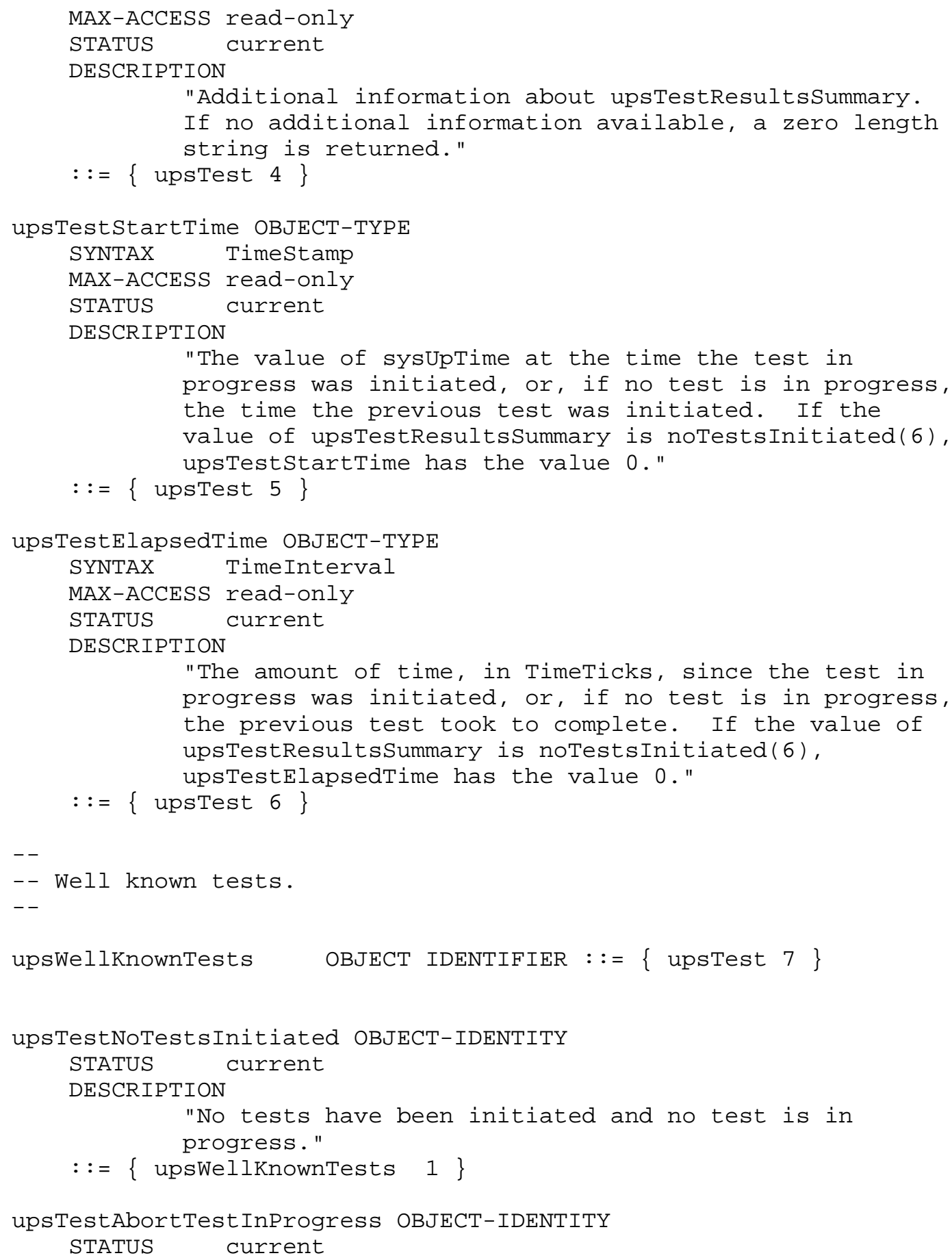




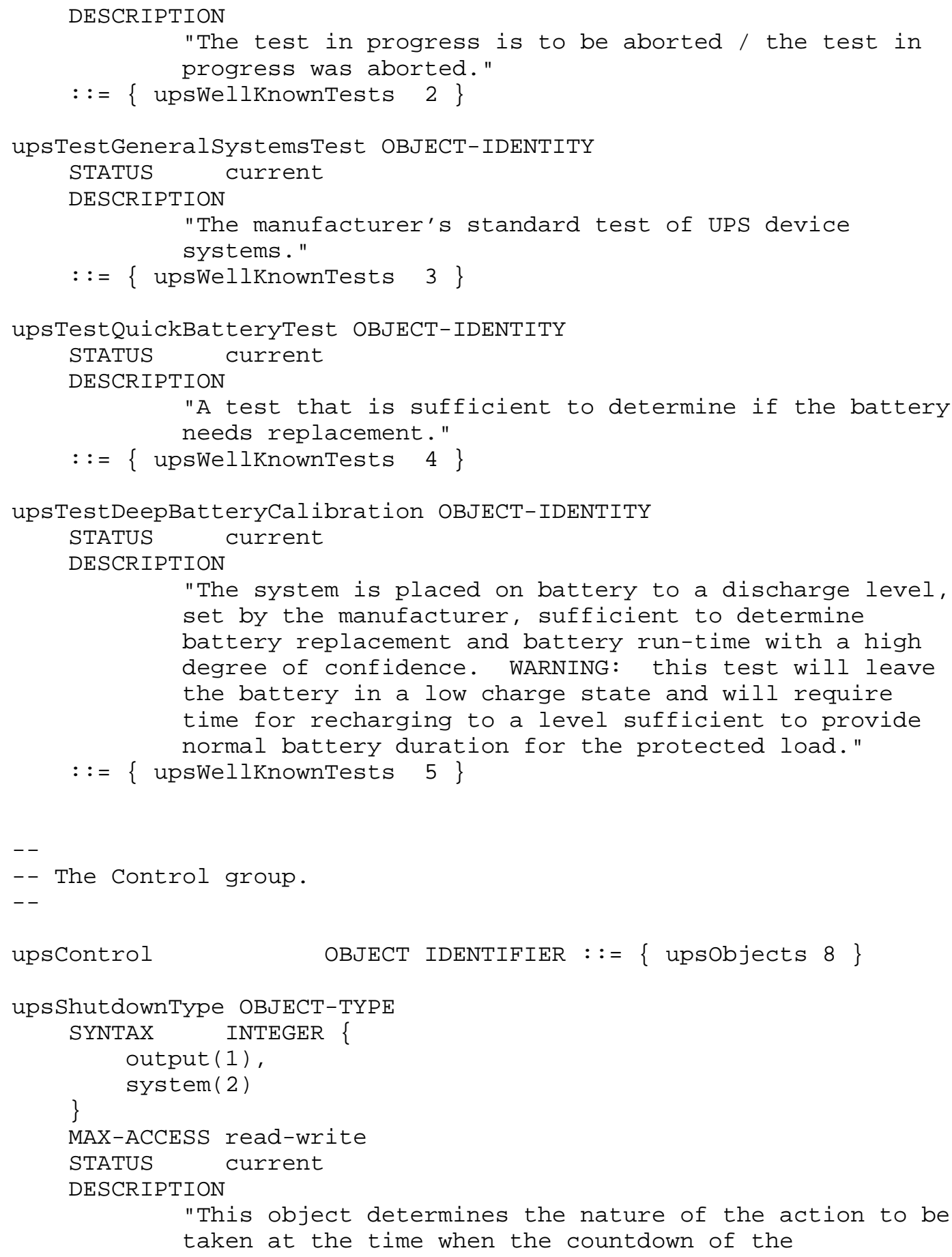




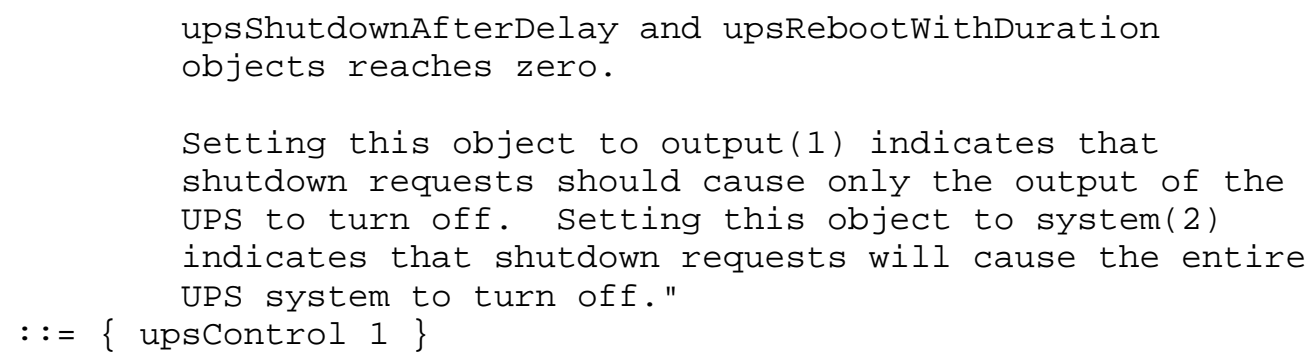


object to -1 will abort the countdown. If the output is already on at the time the countdown reaches 0 , then nothing will happen. Sets to this object override the effect of any upsstartupAfterDelay countdown or upsRebootWithDuration countdown in progress. When read, upsstartupAfterDelay will return the number of seconds until startup, or -1 if no startup countdown is in effect. If the countdown expires during a utility failure, the startup shall not occur until the utility power is restored. On some systems, if the agent is restarted while a startup countdown is in effect, the countdown is aborted."

$::=\{$ upscontrol 3$\}$

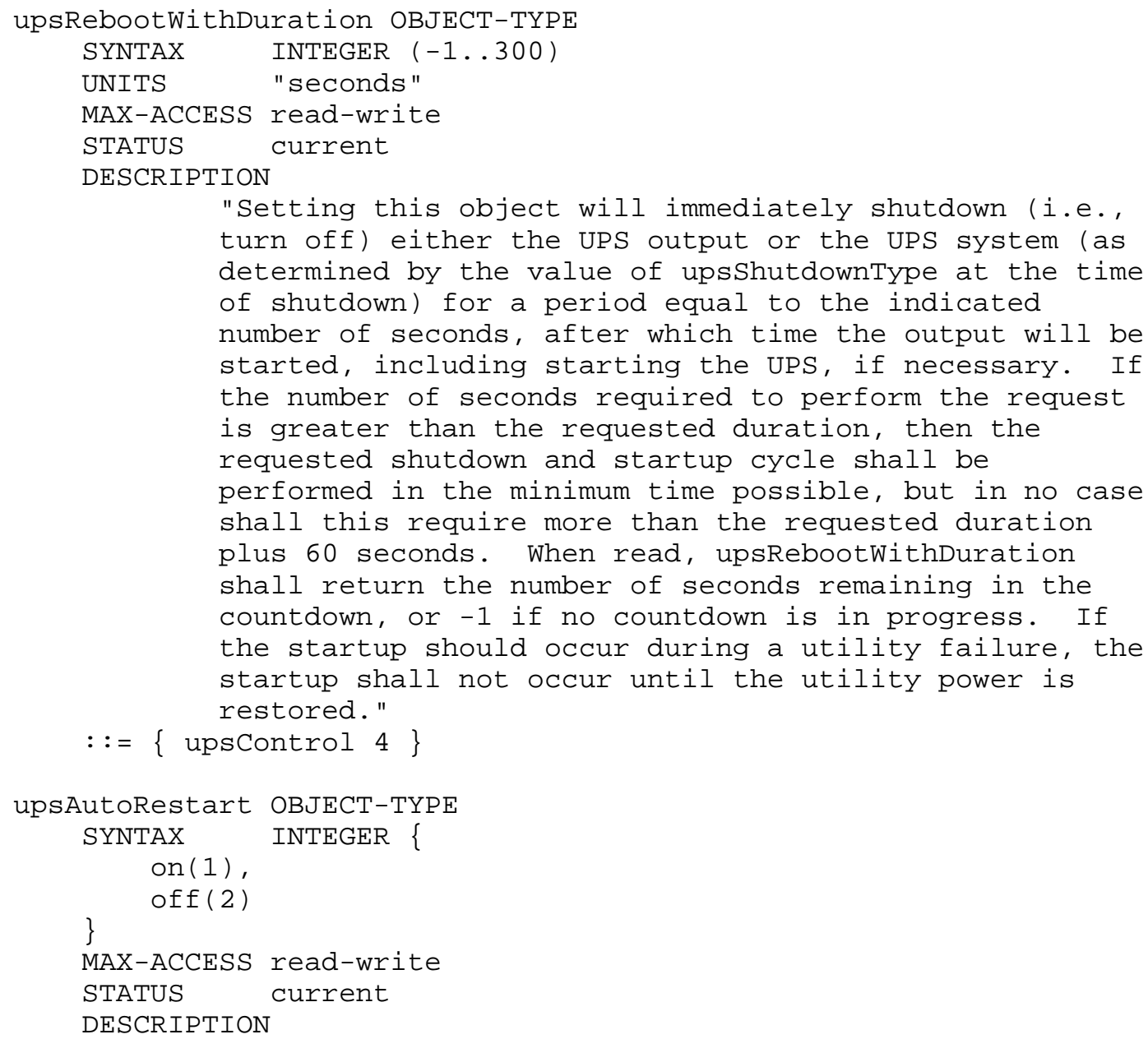




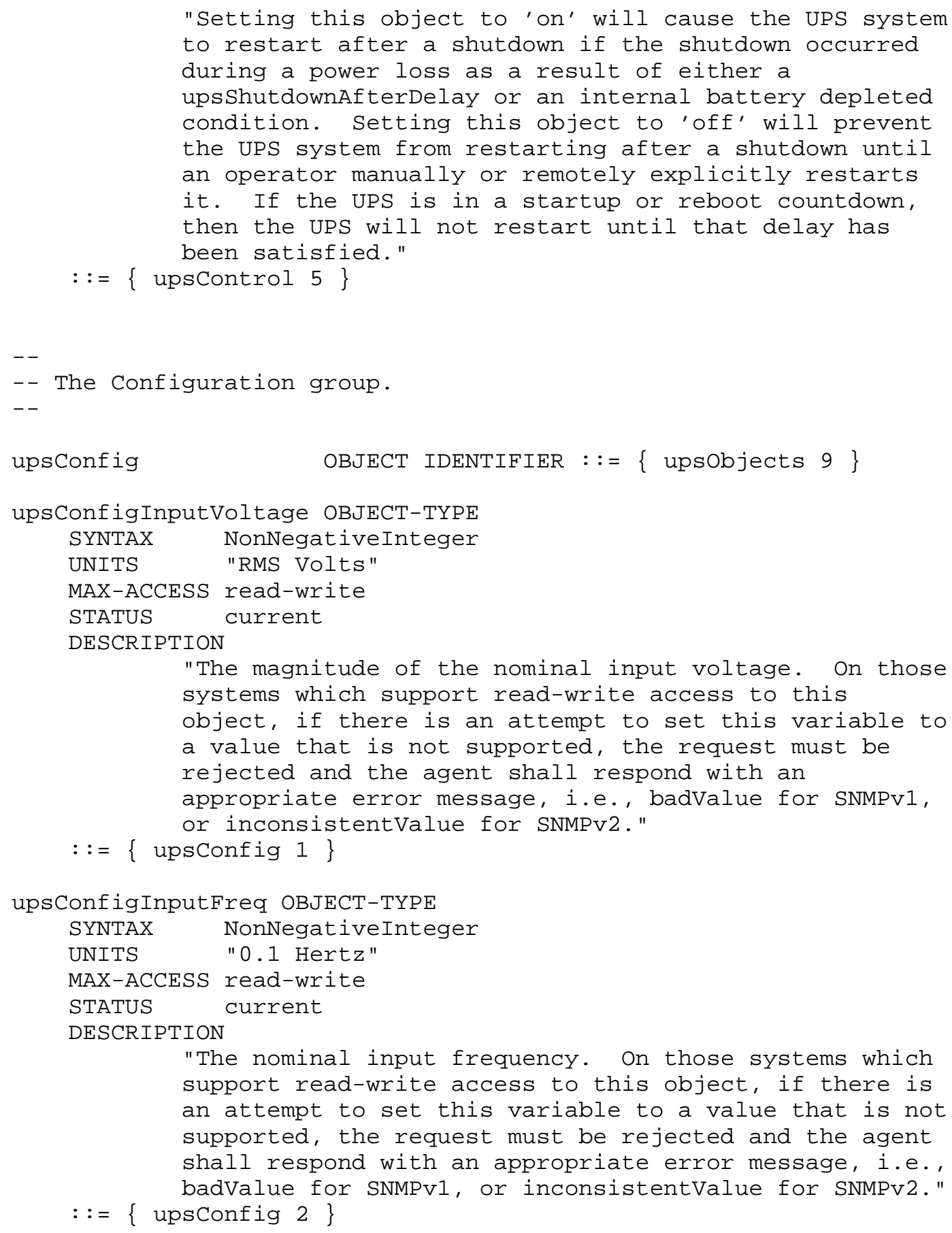




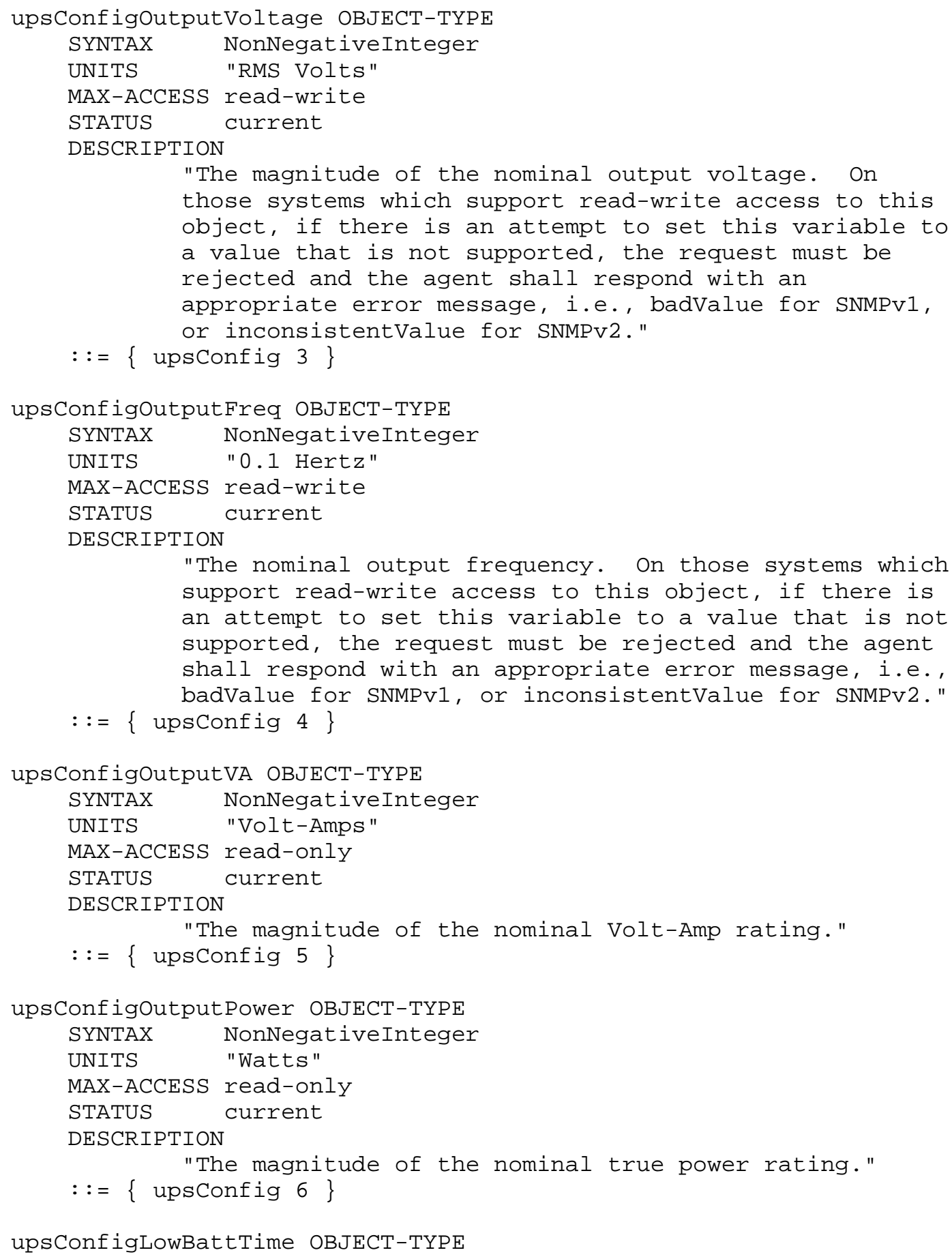




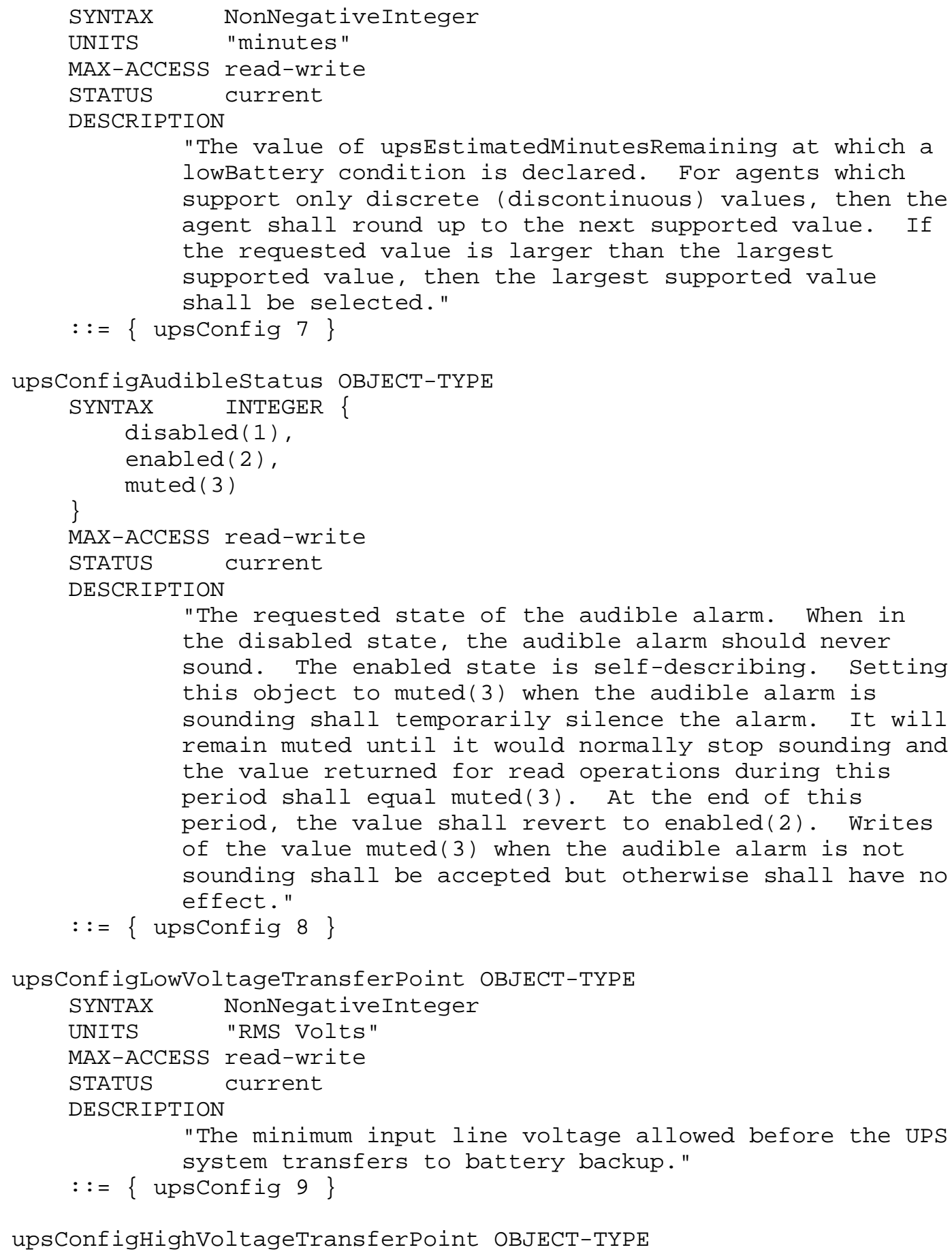




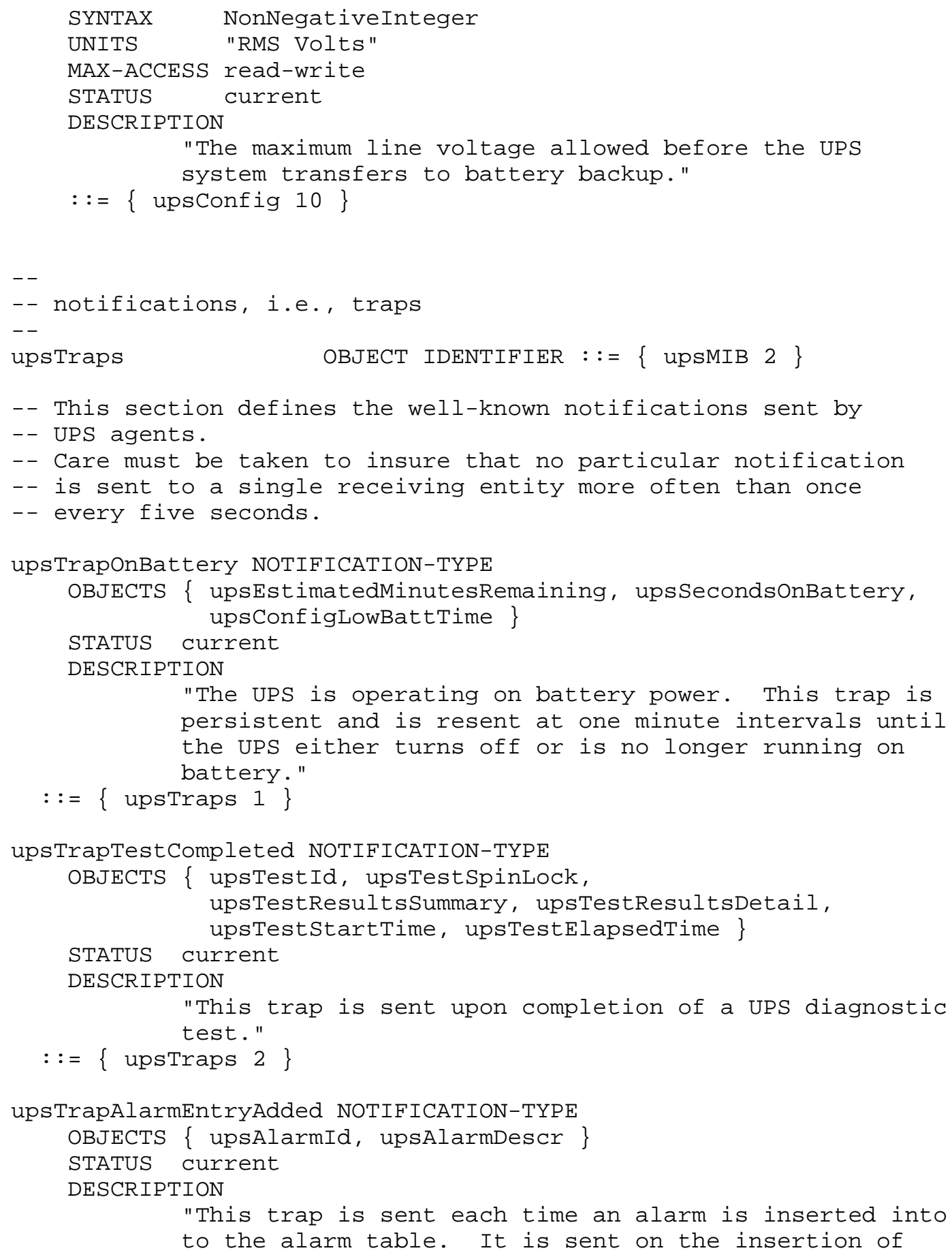




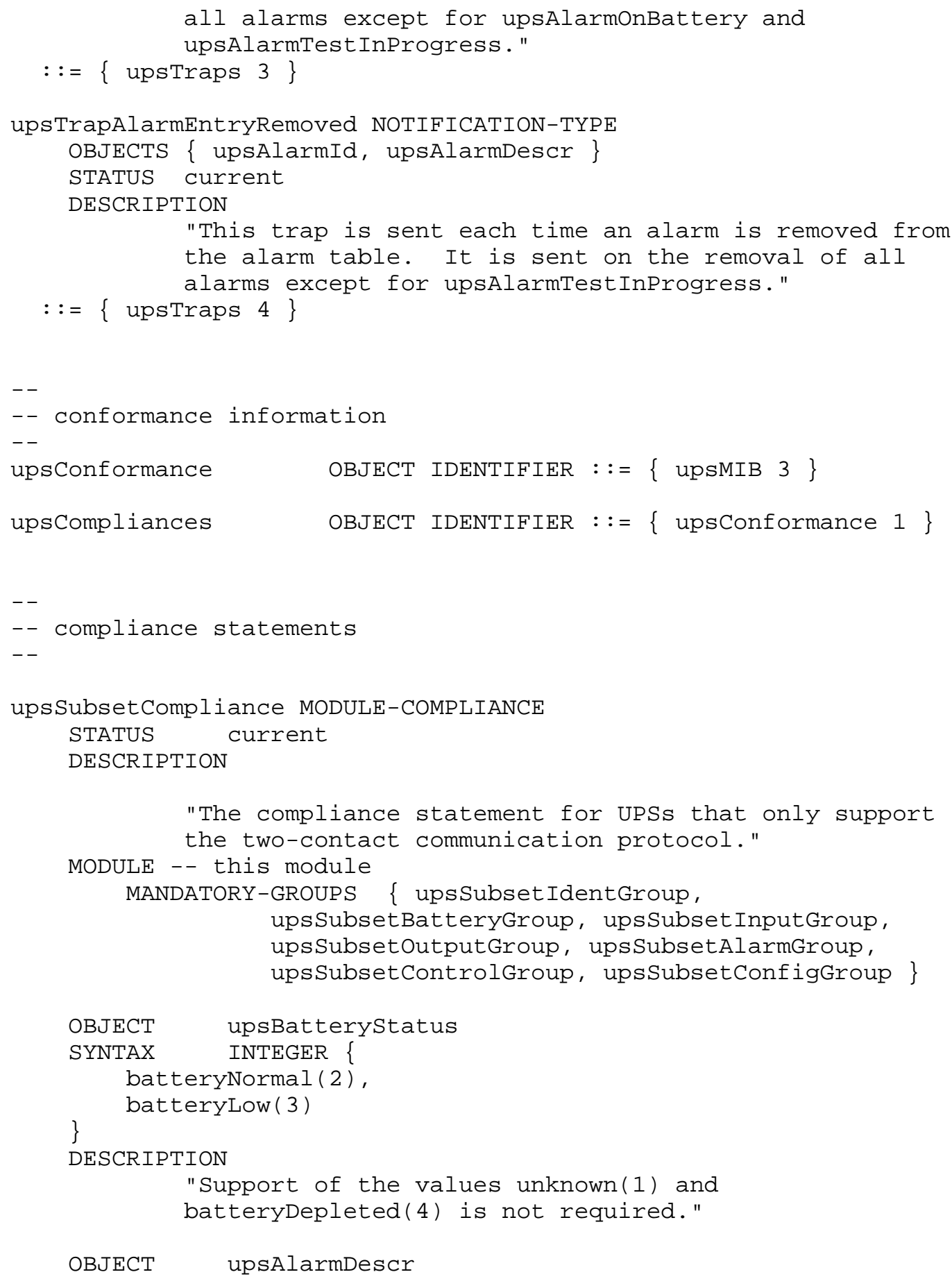




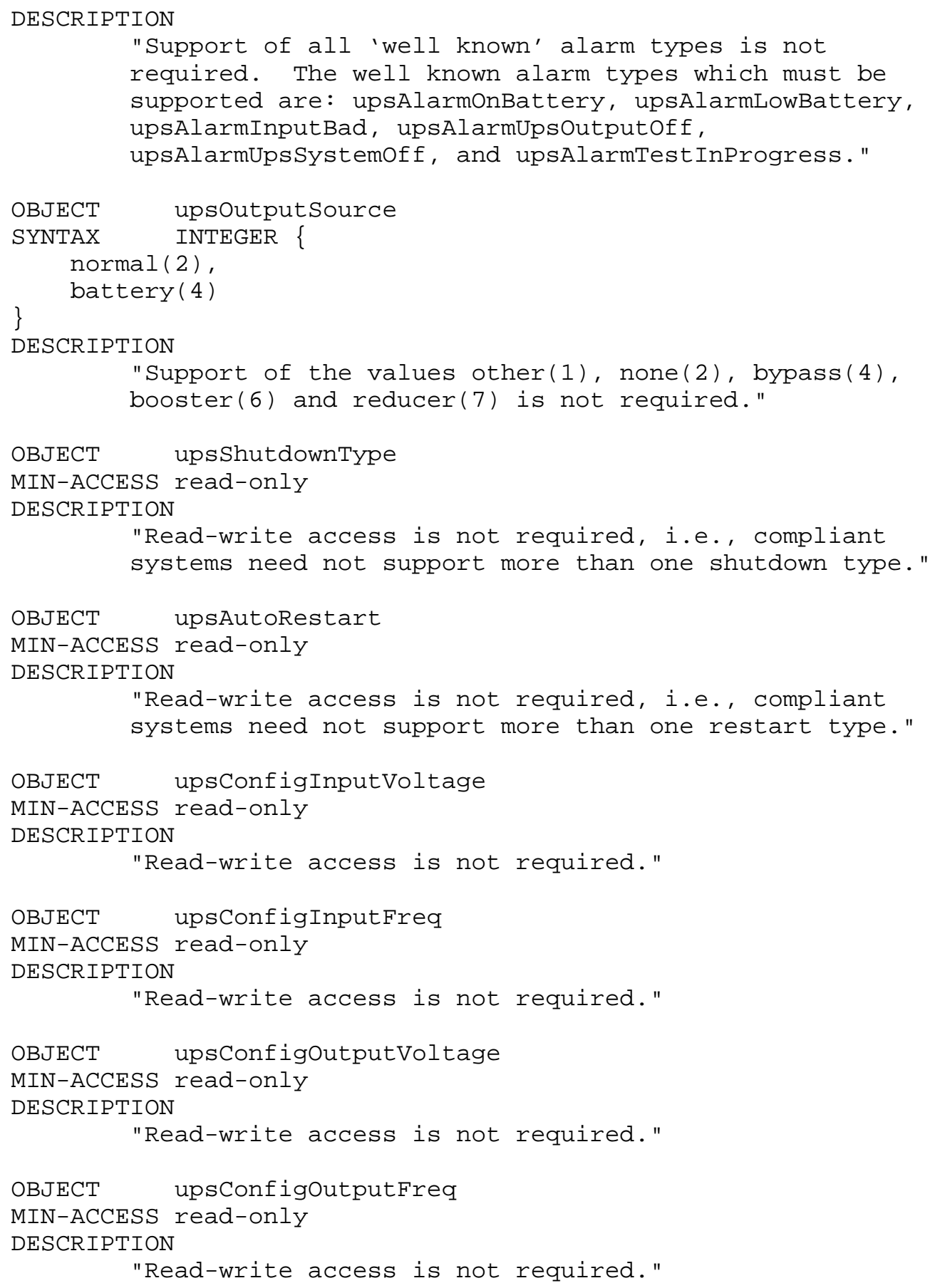


$::=\{$ upscompliances 1$\}$

upsBasicCompliance MODULE-COMPLIANCE

STATUS current

DESCRIPTION

"The compliance statement for UPSs that support

full-featured functions, such as control."

MODULE -- this module

MANDATORY-GROUPS \{ upsBasicIdentGroup,

upsBasicBatteryGroup, upsBasicInputGroup, upsBasicOutputGroup, upsBasicAlarmGroup, upsBasicTestGroup, upsBasicControlGroup, upsBasicConfigGroup \}

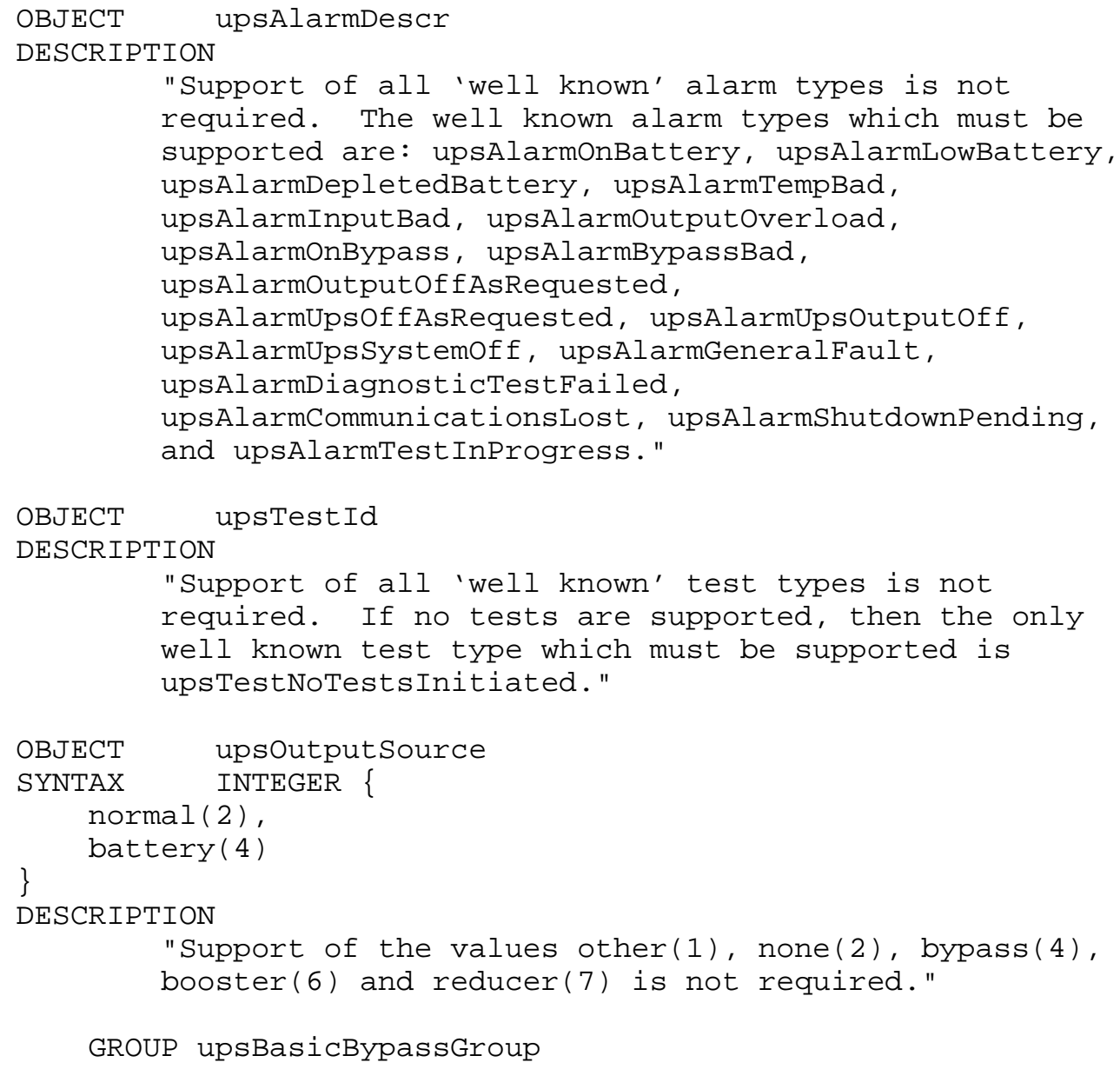




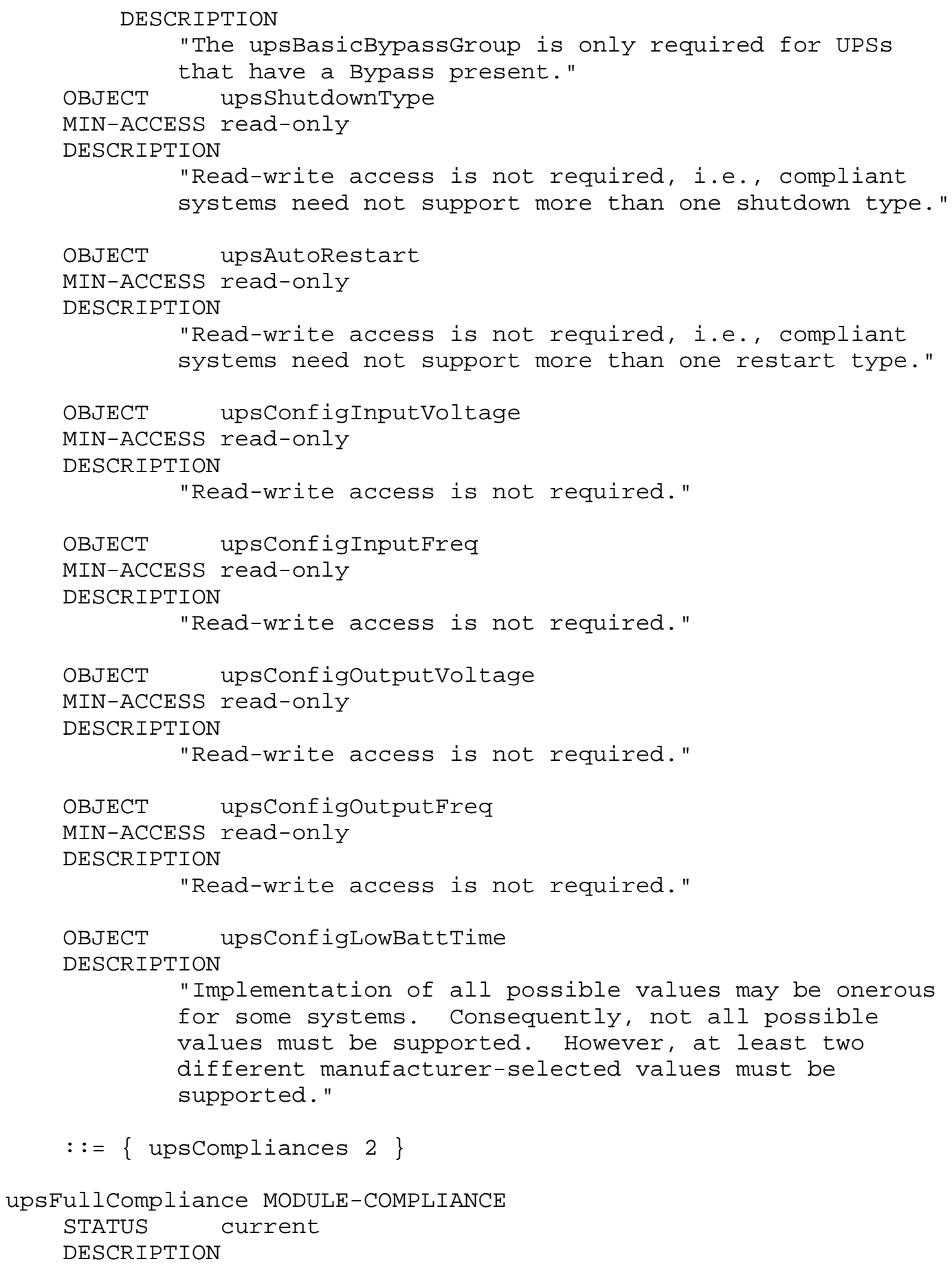




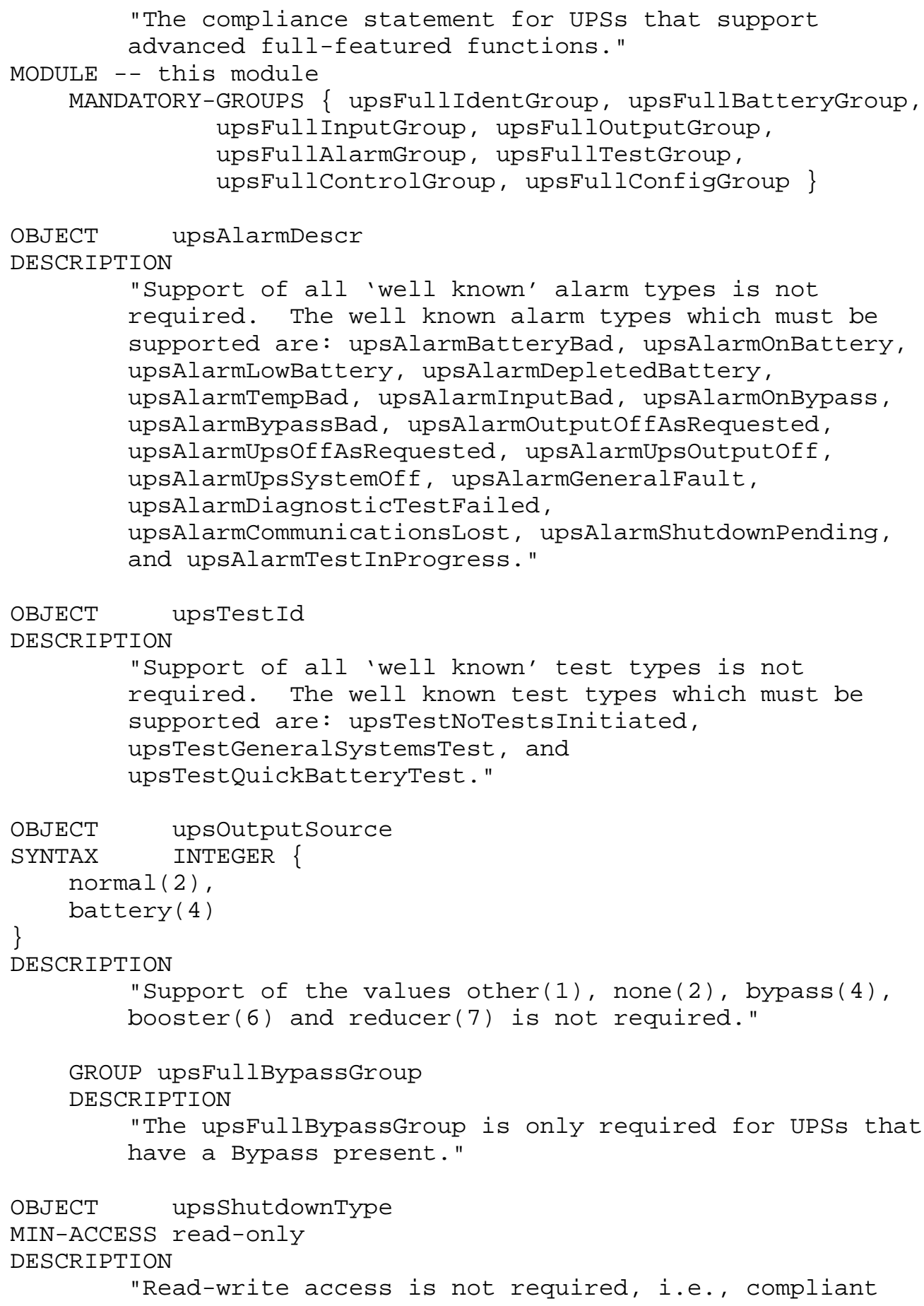




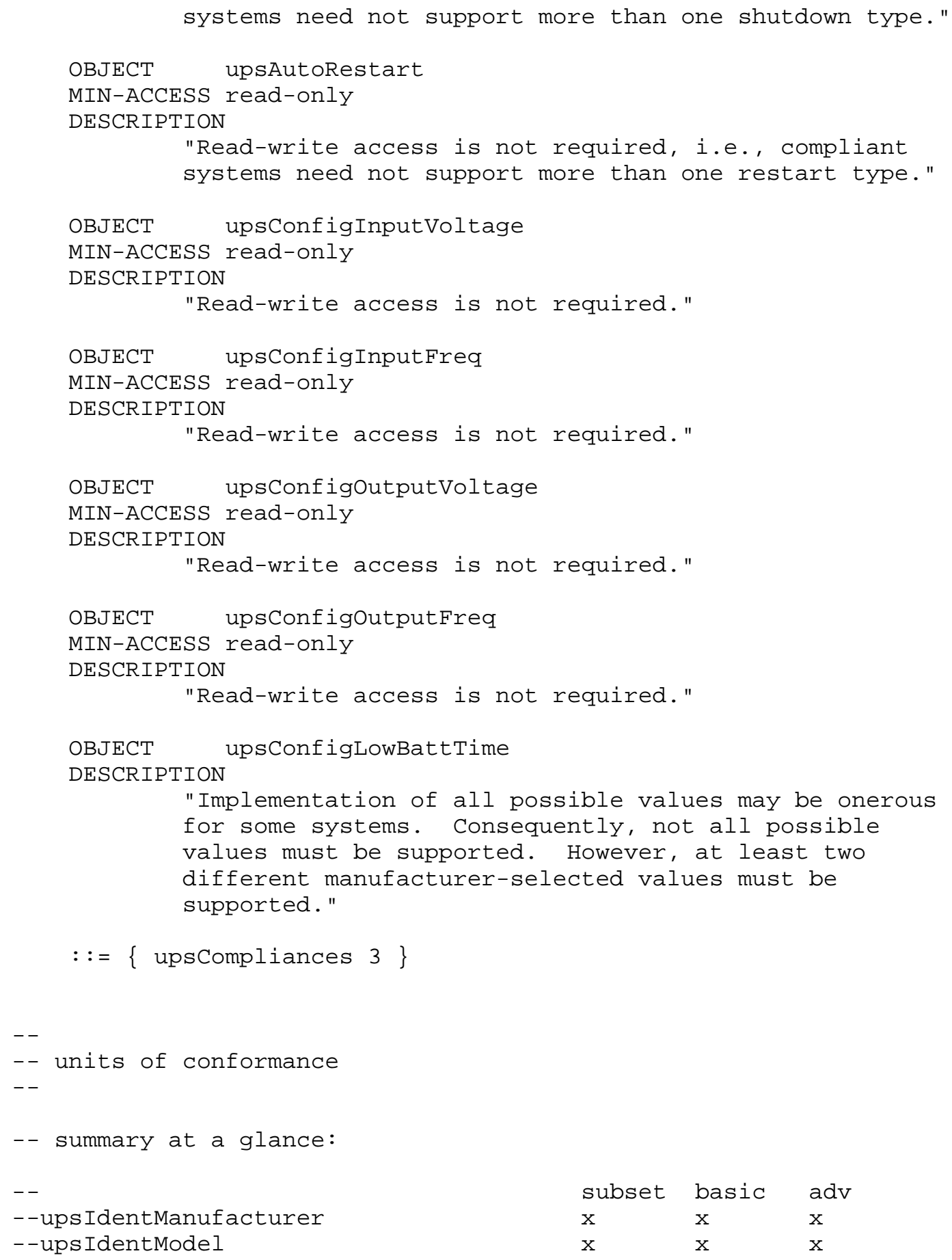




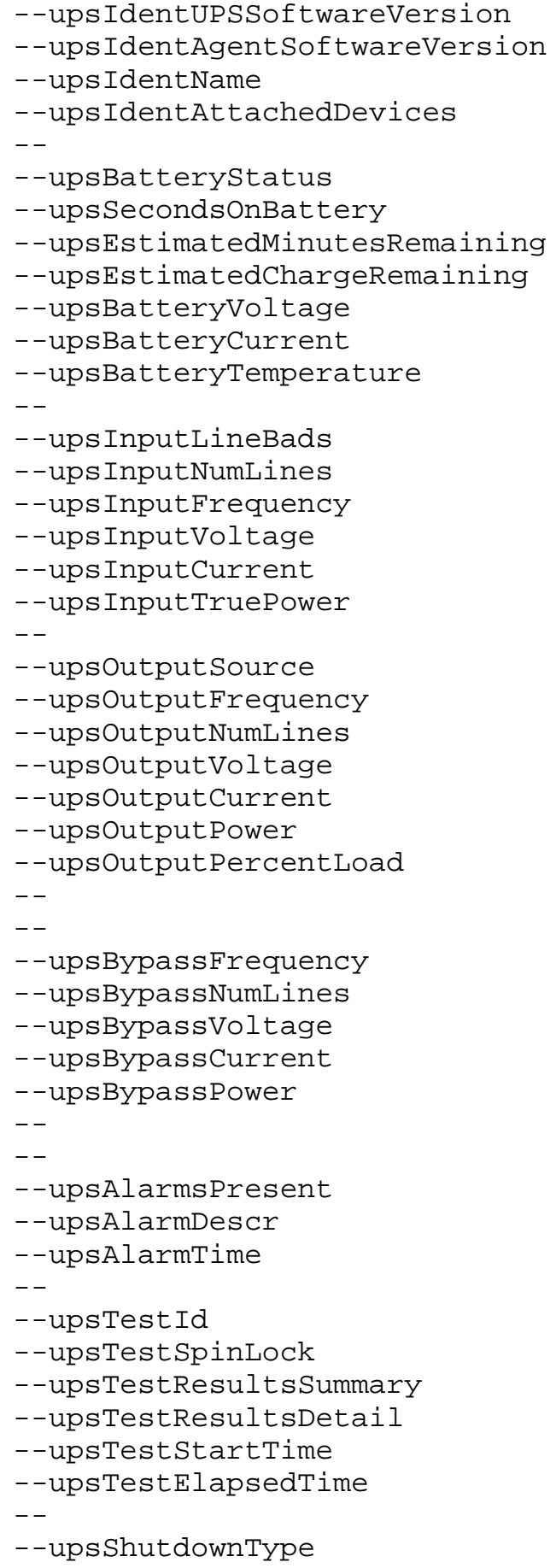

$\begin{array}{llll} & x & x \\ x & x & x & \\ x & x & x & \\ x & & & \\ x & x & x & \text { notes } \\ x & x & x & \\ & & x & \\ & & x & \end{array}$

$\mathrm{x}$

$\begin{array}{cc}x & x \\ x & x \\ x & x \\ x & x\end{array}$

$\mathrm{x}$

$\begin{array}{cc}\mathrm{x} & \mathrm{x} \\ \mathrm{x} & \mathrm{x} \\ \mathrm{x} & \mathrm{x} \\ \mathrm{x} & \mathrm{x} \\ & \mathrm{x} \\ & \mathrm{x} \\ & \mathrm{x}\end{array}$

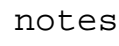
$x$ $x$ $x$ $x$ $\mathrm{x}$ $x \quad x$ $x \quad x$

$x \quad x$

notes $x$

$\mathrm{x}$

$\mathrm{x}$

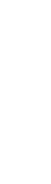

[Page 36] 


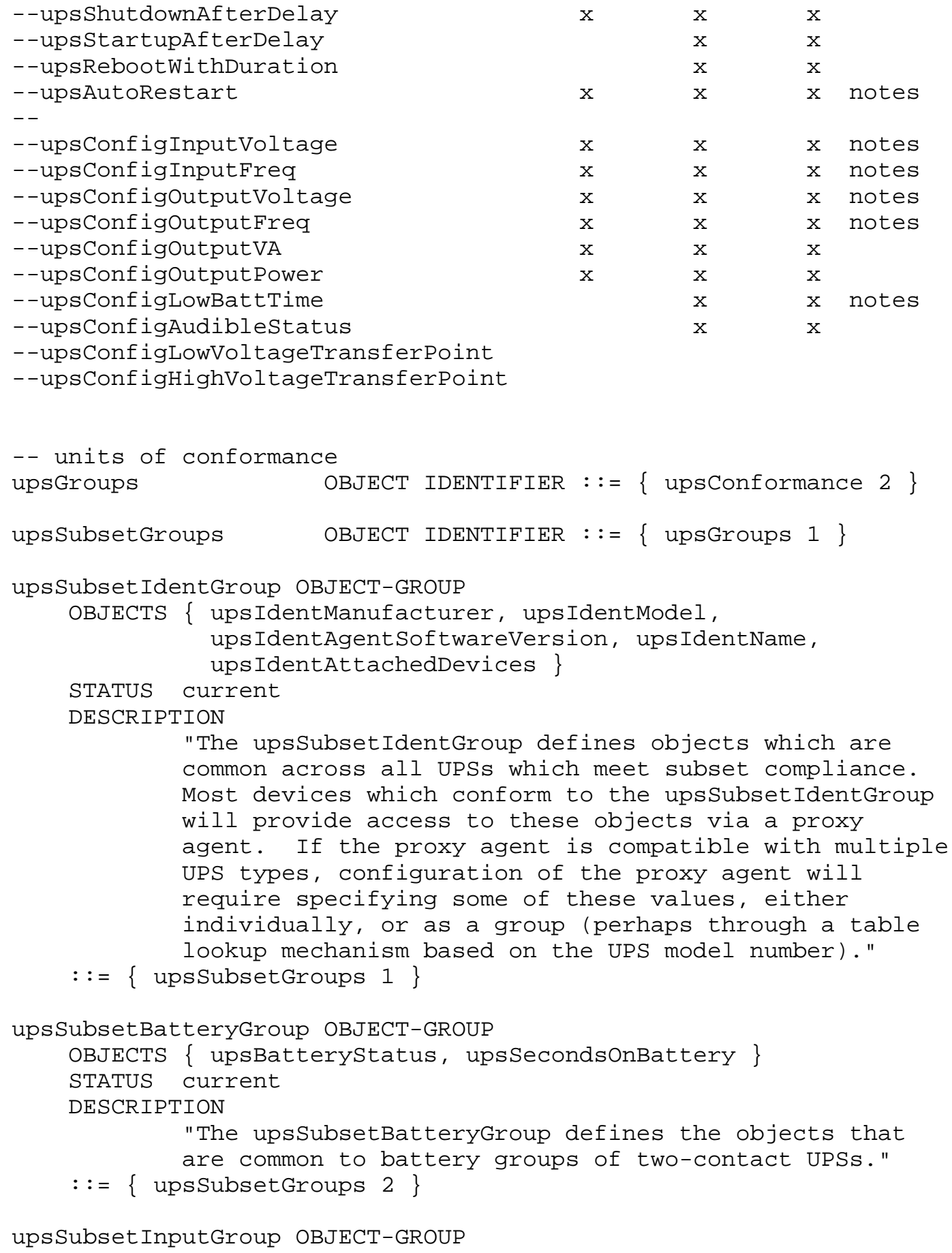




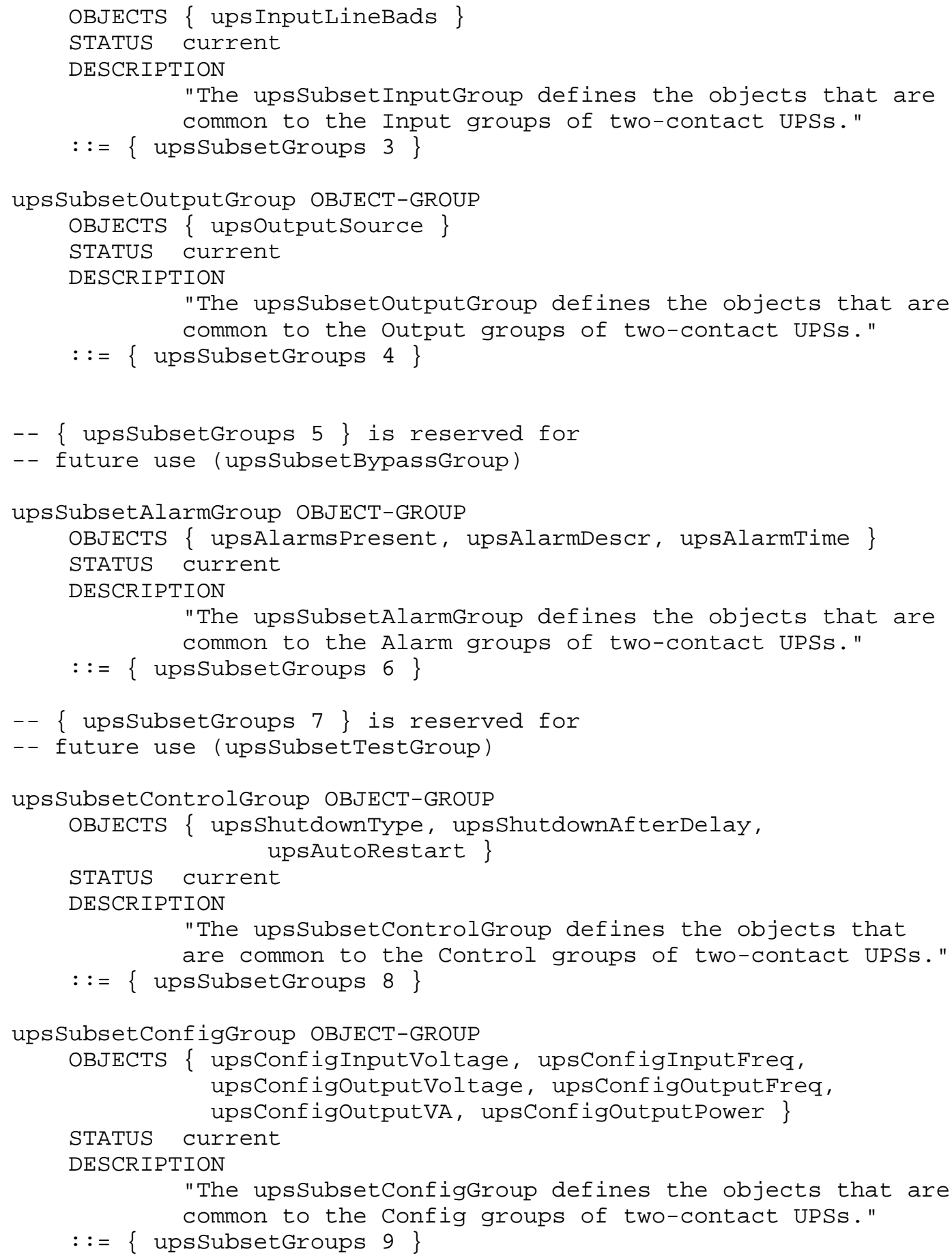




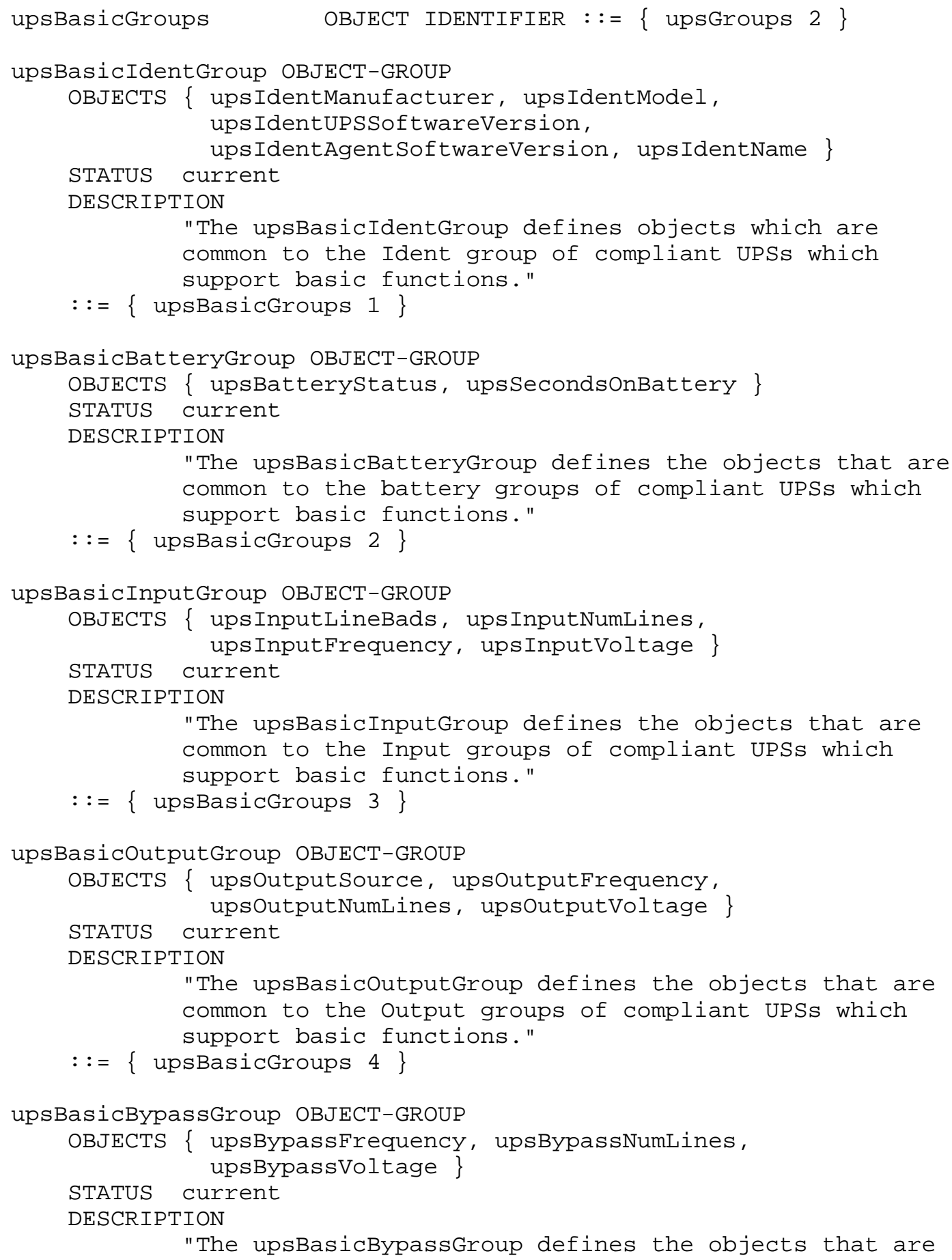




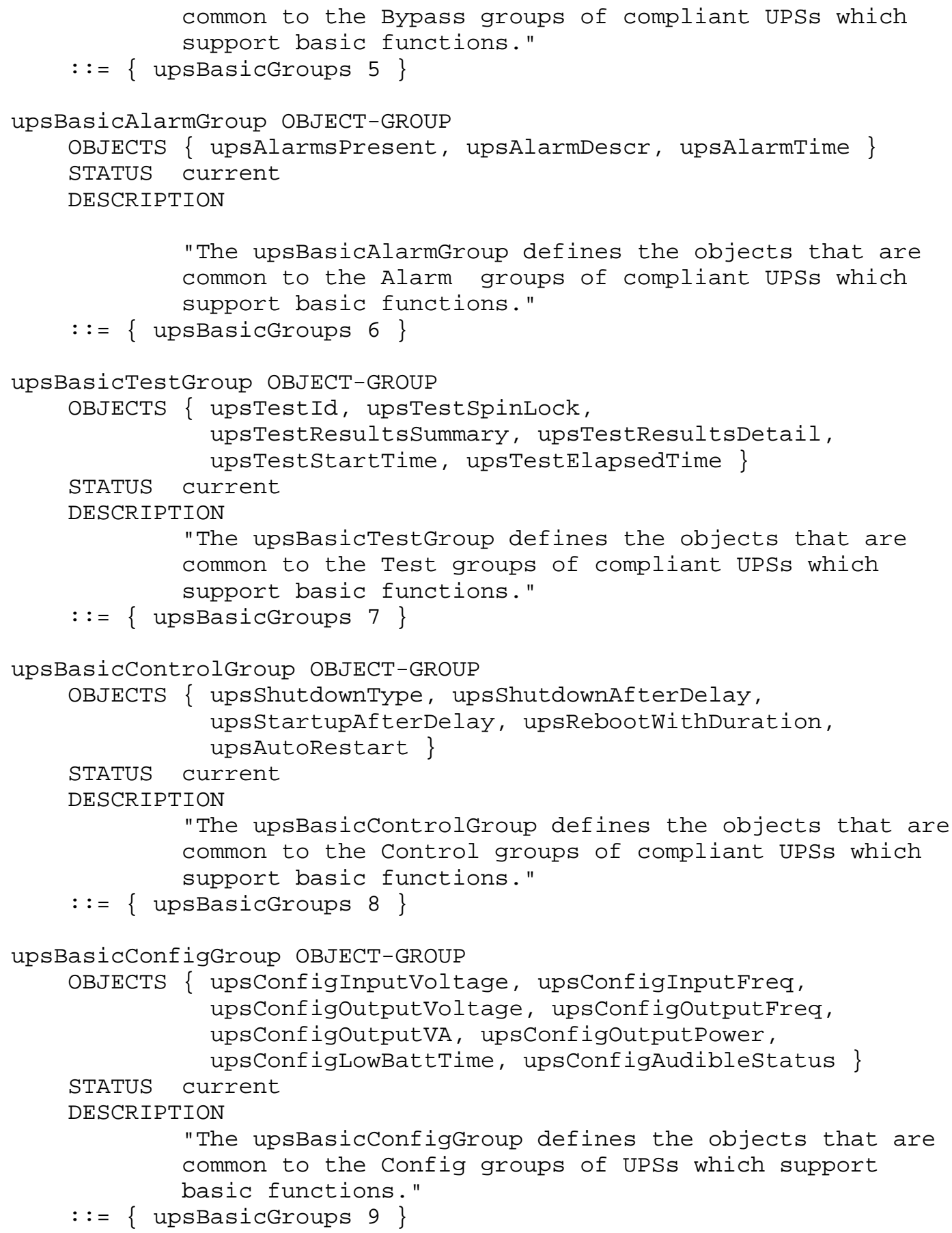




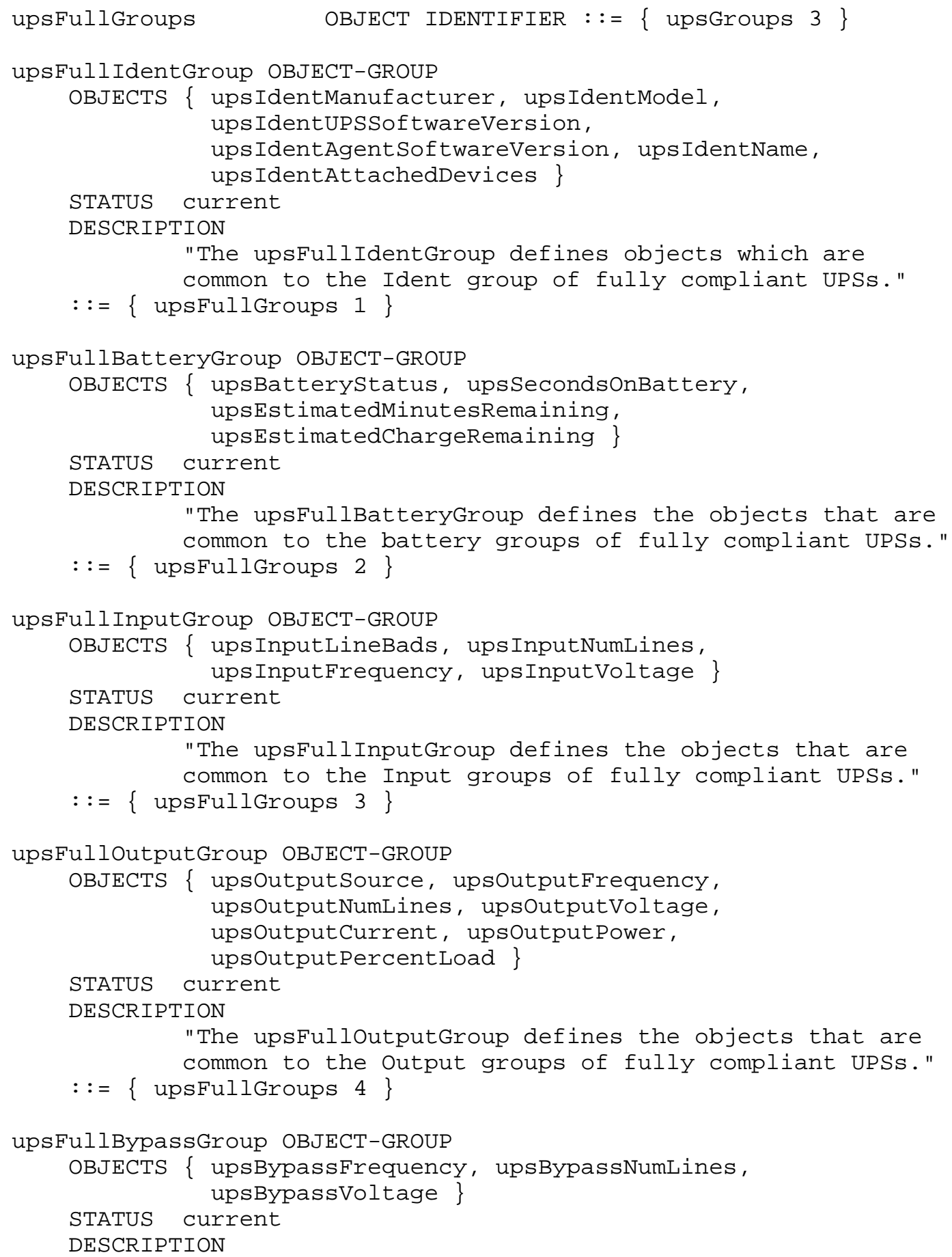




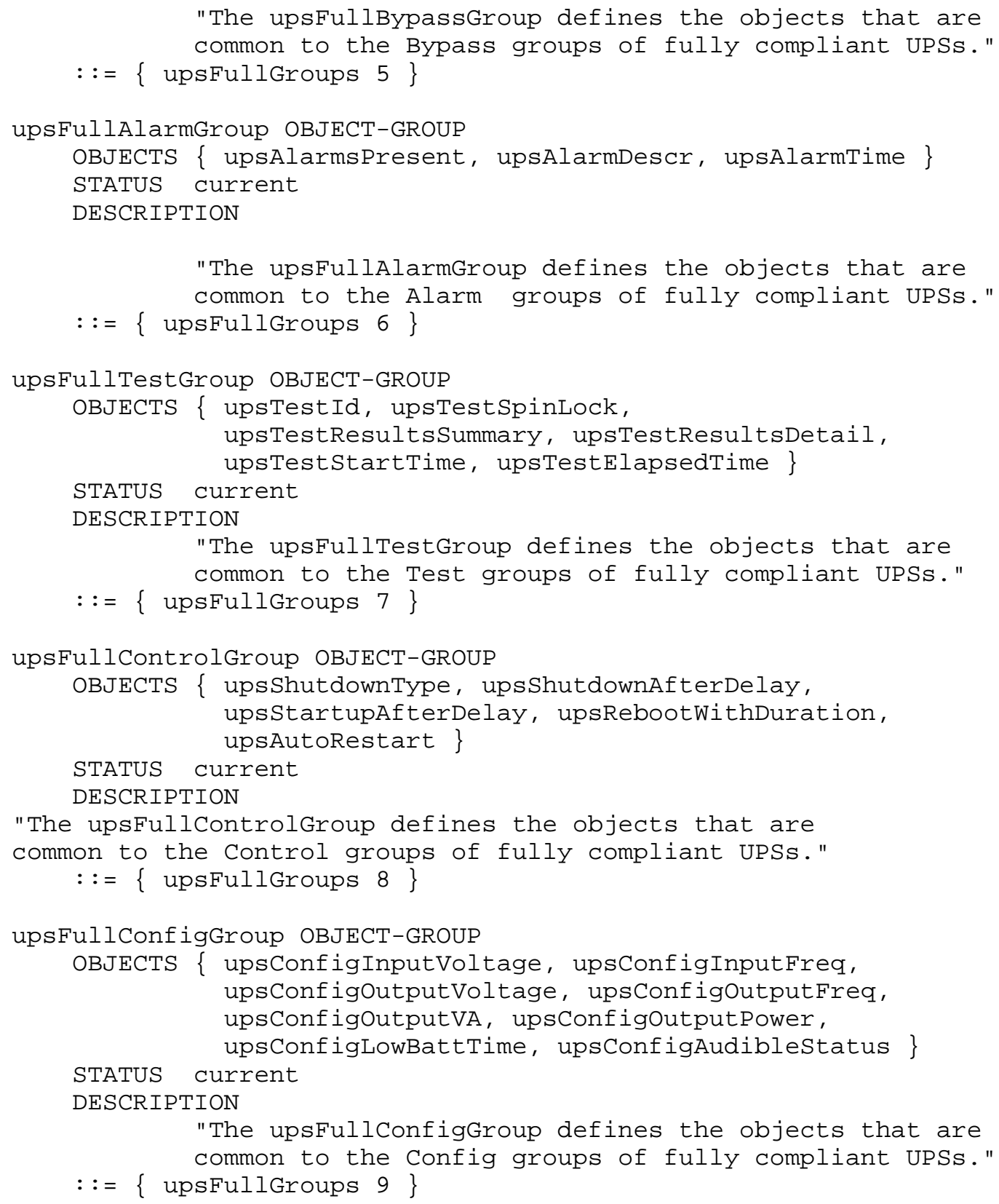

END 


\section{Acknowledgements}

The UPS MIB represents the combined work of the IETF UPS MIB Working Group, with particular, substantial authorship contributions from:

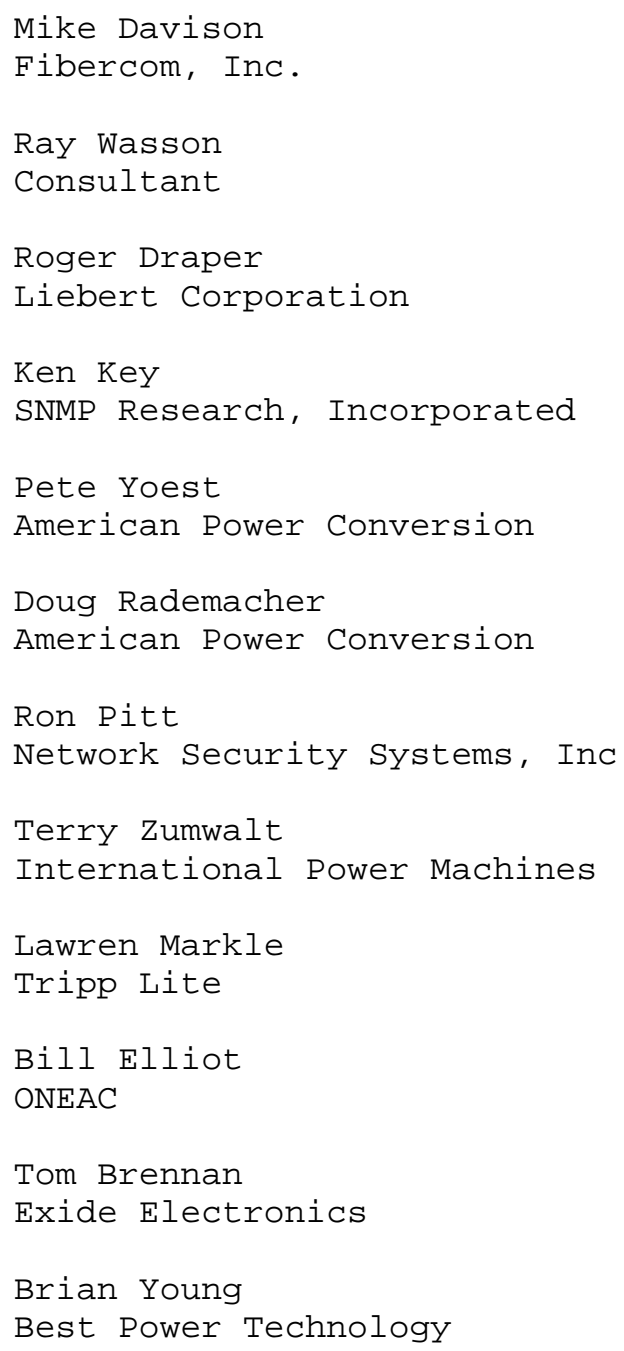




\section{References}

[1] Case, J., Fedor, M., Schoffstall, M., and J. Davin, "Simple Network Management Protocol", STD 15, RFC 1157, SNMP Research, Inc., Performance Systems International, Performance systems International, MIT Laboratory for Computer Science, May 1990.

[2] Case, J., McCloghrie, K., Rose, M., and S. Waldbusser, "Protocol Operations for Version 2 of the Simple Network Management Protocol (SNMPv2)", RFC 1448, SNMP Research, Inc., Hughes LAN Systems, Dover Beach Consulting, Inc., Carnegie Mellon University, April 1993.

[3] McCloghrie, K., and M. Rose, Editors, "Management Information Base for Network Management of TCP/IP-based internets: MIB-II", STD 17, RFC 1213, Hughes LAN Systems, Performance systems International, March 1991.

[4] Case, J., McCloghrie, K., Rose, M., and S. Waldbusser, "Structure of Management Information for Version 2 of the Simple Network Management Protocol (SNMPV2)", RFC 1442, SNMP Research, Inc., Hughes LAN Systems, Dover Beach Consulting, Inc., Carnegie Mellon University, April 1993.

[5] Case, J., McCloghrie, K., Rose, M., and S. Waldbusser, "Conformance Statements for Version 2 of the Simple Network Management Protocol (SNMPV2)", RFC 1444, SNMP Research, Inc., Hughes LAN Systems, Dover Beach Consulting, Inc., Carnegie Mellon University, April, 1993.

[6] Case, J., McCloghrie, K., Rose, M., and S. Waldbusser, "Textual Conventions for Version 2 of the Simple Network Management Protocol (SNMPV2)", RFC 1443, SNMP Research, Inc., Hughes LAN Systems, Dover Beach Consulting, Inc., Carnegie Mellon University, April 1993. 
7. Security Considerations

Security issues are not discussed in this memo.

8. Author's Address

Jeffrey D. Case, Ph.D.

SNMP Research, Incorporated

3001 Kimberlin Heights Road

Knoxville, Tennessee 37920

Phone: (615) 573-1434

EMail: case@SNMP.COM 